\title{
Genome-wide characterization and expression analyses of superoxide dismutase $(S O D)$ genes in Gossypium hirsutum
}

\author{
Wei Wang ${ }^{\dagger}$, Xiaopei Zhang ${ }^{\dagger}$, Fenni Deng, Rui Yuan and Fafu Shen ${ }^{*}$
}

\begin{abstract}
Background: Superoxide dismutases (SODs) are a key antioxidant enzyme family, which have been implicated in protecting plants against the toxic effects of reactive oxygen species. Despite current studies have shown that the gene family are involved in plant growth and developmental processes and biotic and abiotic stress responses, little is known about its functional role in upland cotton.

Results: In the present study, we comprehensively analyzed the characteristics of the SOD gene family in upland cotton (Gossypium hirsutum). Based on their conserved motifs, 18 GhSOD genes were identified and phylogenetically classified into five subgroups which corroborated their classifications based on gene-structure patterns and subcellular localizations. The GhSOD sequences were distributed at different densities across 12 of the 26 chromosomes. The conserved domains, gene family evolution cis-acting elements of promoter regions and miRNA-mediated posttranscriptional regulation were predicted and analyzed. In addition, the expression pattern of 18 GhSOD genes were tested in different tissues/organs and developmental stages, and different abiotic stresses and abscisic acid, which indicated that the SOD gene family possessed temporal and spatial specificity expression specificity and may play important roles in reactive oxygen species scavenging caused by various stresses in upland cotton.
\end{abstract}

Conclusions: This study describes the first genome-wide analysis of the upland cotton SOD gene family, and the results will help establish a foundation for the further cloning and functional verification of the GhSOD gene family during stress responses, leading to crop improvement.

Keywords: SOD, Upland cotton, Genome wide analysis, Abiotic stress, Expression profiles

\section{Background}

Allotetraploid upland cotton (Gossypium hirsutum) accounts for more than $90 \%$ of cultivated cotton worldwide and is an important economic crop that provides fiber, seed oil, and protein meal [1]. However, its growth and yield are affected by various environmental stressors, including salinity, drought, heat, cold, herbicides, heavy metals and pathogens. The most common result of such stress is the generation of toxic reactive

\footnotetext{
*Correspondence: ffshen@sdau.edu.cn

${ }^{\dagger}$ Equal contributors

State Key Laboratory of Crop Biology, College of Agronomy, Shandong

Agricultural University, Tai'an 271018, Shandong, People's Republic of China
}

oxygen species (ROS). Excess ROS, such as superoxide anion, hydroxyl radical, hydrogen peroxide and singlet oxygen, could result in membrane damage, protein oxidation and DNA lesions, and could even lead to irreparable metabolic dysfunctions and cell death. To cope with ROS toxicity, plants have developed efficient antioxidative mechanisms, including many non-enzymatic and enzymatic defense systems. Among the enzymatic defense systems, superoxide dismutases (SODs) (EC 1.15.1.1), a family of antioxidant enzymes, are the first line of defense against oxidative damage and are ubiquitous in every cell of all plant types. As a major defense system against oxidative stress in plants, SOD catalyzes the conversion or 
dismutation of toxic superoxide anion radicals to hydrogen peroxide and molecular oxygen [2].

In plants, SODs have been classified into three groups based on the type of prosthetic metal: copper/zinc $(\mathrm{Cu} /$ $\mathrm{Zn}$ )-SOD, manganese ( $\mathrm{Mn}$ )-SOD and iron (Fe)-SOD. SOD proteins are encoded by nuclear genes and distributed to different cellular compartments. $\mathrm{Cu} / \mathrm{Zn}$-SOD is present chiefly in chloroplasts and in the cytosol and mitochondria. Mn-SOD is mainly localized in mitochondria but is also in different types of peroxisomes. FeSOD occurs in chloroplasts, and also in peroxisomes and mitochondria [3]. A considerable number of $S O D$ genes have been cloned from various monocots and dicots [4-6], and, since the first $S O D$ gene cloned from maize [7], reports have indicated that SODs are important for stress tolerance [8,9]. Additionally, plants with SOD gene families that have been characterized at the genome-wide level include Arabidopsis thaliana [10], Dimocarpus longan [4], Sorghum bicolor [11], Populus trichocarpa [5], Musa acuminate [6], Gossypium raimondii and Gossypium arboretum [12], and the numbers of each of the three SOD-type genes vary among them.

In a previous study, Voloudakis et al. studied the SOD isoenzymes in upland cotton using genetic engineering methods and revealed that the three SOD isoenzymes respond to bacterial blight of cotton [13]. Additionally, Holaday et al. suggested that Mn-SOD increases the tolerance of cotton to low temperature and high light using transgenic technology [14]. However, these studies focused only on the proteins and activity changes, and were unable to effectively elucidate the exact roles of the cotton SOD gene family under adverse conditions. Recently, the whole-genome sequences of two diploid cottons (G. raimondii and G. arboretum) [15-17] and two allotetraploid cottons (G. hirsutum and Gossypium barbadense) [18-21] were made available to the public, facilitating molecular studies on the expression and the regulatory mechanisms of the cotton $S O D$ gene family in response to various stresses. Using these genomes, a genome-wide analysis of the $S O D$ gene family in two diploid cottons was performed. In this study, the identification of the $S O D$ gene family in upland cotton (G. hirsutum) was performed to analyze genomic organization, gene structure, motif composition and phylogenetic relationships. Then, the putative promoters of the upland cotton $S O D$ s were also investigated, ciselements involved in stress responses were analyzed, and miRNA target sites of GhSODs were predicted to further clarify the regulatory mechanisms of gene expression. Finally, we studied the expression patterns of the upland cotton $S O D$ gene family under abiotic (salt, drought, cold and heat) and hormonal stresses [abscisic acid (ABA)] using a real-time quantitative PCR (qPCR) detection system. This was the first comprehensive study of the $S O D$ gene family in upland cotton and provided valuable information for understanding the classification, evolution and putative functions of this family on the whole-genome scale.

\section{Methods \\ Identification of SOD genes}

The G. hirsutum genomes and annotation files [Gossypium hirsutum (AD1) Genome NAU-NBI Assembly v1.1 \& Annotation v1.1] were downloaded from CottonGen (https://www.cottongen.org). We then filtered gene annotation results based on the following criteria [22]: (1) the longest transcript in each gene loci was chosen to represent that locus; (2) CDSs with length $<150$ bp were filtered out; (3) CDSs with percentages of ambiguous nucleotides ('N') $>50 \%$ were filtered out; (4) CDSs with internal termination codons were filtered out; and (5) the CDSs with hits (BLAST identities $\geq 80 \%$ ) to RepBase sequences were filtered out (http://www.girinst.org/repbase/index.html). To identify members of the $S O D$ gene family in G. hirsutum, we used SOD data from previous studies in G. raimondii and $G$. arboretum [12] and retrieved SOD protein sequences from the NCBI (http://www.ncbi.nlm.nih.gov/protein/) and the JGI database (http://www.phytozome.net). The full-length protein sequences from $A$. thaliana (NCBI accession: NP_172360.1, NP_565666.1, NP_197311.1, NP_199923.1, NP_197722.1 and NP_187703.1) were used as query sequences to perform multiple database searches using the BLAST algorithm for Proteins (BLASTP) [23]. After removing alignments with identities $<50 \%$, the resulting candidate SOD proteins were aligned to each other to ensure that no gene was represented multiple times. InterProScan (version 4.8) [24] was further used to confirm the inclusion of the SOD domain in each candidate sequence using the Pfam database. The $S O D$ gene members of the other 17 plant genomes shown in Additional file 1 were identified using similar methods said above.

SOD gene data, including accession number, chromosomal location and ORF length, were collected from the G. hirsutum genome and annotation files. All of the candidate SOD protein sequences were analyzed using InterProScan (version 4.8) with the Pfam database [25], and conserved domains were located with Pfam HMM search (http://pfam.xfam.org/search). Conserved protein motifs were predicted by MEME Suite (http://meme-suite.org/ tools/meme) with the default settings, except that the minimum and maximum motif widths were set to 20 and 150 amino acids [26]. Physico-chemical characteristics of SOD proteins, including the number of amino acids, molecular weight, theoretical isoelectric point and instability index, were calculated using the ProtParam tool (http://www.expasy.org/tools/protparam.html). Predictions of subcellular localizations of SOD proteins were 
performed with CELLO v.2.5 (http://cello.life.nctu.edu.tw/) [27] and WoLF PSORT servers (http://www.genscript. com/wolf-psort.html) [28].

\section{Phylogenetic analysis}

We aligned the full-length coding sequences of plant $S O D$ genes using the ClustalW program with default parameters [29]. The Gblocks_0.91b local program selected the conserved blocks from multiple alignments [30]. ModelGenerator_0.85 was used to evaluate the fit of major models of amino acid substitutions, and the Bayesian information criterion and Akaike information criterion were applied to select the fit model that met the amino acid frequencies and rates of amino acid substitutions for each amino acid pair using a discrete gamma distribution [31]. The phylogenetic tree was then constructed using the maximum likelihood method with a bootstrap analysis of 1,000 replicates and the Jones-Taylor-Thornton (JTT) with Gamma Distributed (G) substitution model using MEGA6.0 software [32].

\section{Chromosomal locations and syntenic analysis}

The chromosomal distribution of $S O D$ genes was drafted from top to bottom on upland cotton chromosomes according to gene positions in the genome annotation by Circos-0.69 (http://circos.ca/) [33]. A syntenic analysis was conducted locally using a method similar to that developed for the Plant Genome Duplication Database (http://chibba.pgml.uga.edu/duplication/) [34]. We used BLAST version 2.2.9 [35] for the pairwise comparison of the filtered SOD protein sets of G. hirsutum, G. raimondii and G. arboreum. Then, MCscanX [36] was employed to identify homologous regions, and syntenic blocks were evaluated using Circos-0.69 (http://circos.ca/) [33]. Default parameters were used in all of the steps. Tandem duplications were characterized as multiple genes of one family located within the same or neighboring intergenic region [37].

\section{Gene sequences and putative functional analysis}

Exons-intron structures of $S O D$ genes from G. hirsutum were identified using GSDS 2.0 (http://gsds.cbi.pku.edu.cn/) [38]. Functional annotations of GhSOD genes were analyzed using the GO term analysis tool (http:// geneontology.org/) [39], based on their molecular functions, biological processes, and cellular localizations. The GO annotation results were plotted by the WEGO web tool (http://wego.genomics.org.cn/ cgi-bin/wego/index.pl) [40].

\section{Prediction of GhSODs regulatory elements}

We obtained cotton miRNA sequences from miRBase (http://www.mirbase.org/) [41], the Plant MicroRNA database (http://bioinformatics.cau.edu.cn/PMRD/) [42], the Cotton EST database (http://www.ncbi.nlm.nih.gov/ nucest) and published articles. GhSOD genes targeted by miRNAs were predicted by searching 5' and 3' UTRs and the CDS of all GhSOD genes for complementary sequences of the cotton miRNAs using the psRNATarget server with default parameters (http://plantgrn.noble. org/psRNATarget/?function=3) (Additional file 2) [43]. We selected the targeted sites with high degrees of complementarity shown in Fig. 6. Transcriptional response elements of $S O D$ gene promoters were predicted using the PlantCARE serve, a database of plant cis-acting regulatory elements and a portal to tools for in silico analysis of promoter sequences (http://bioinformatics.psb.ugent.be/ webtools/plantcare/html/) [44].

\section{RNA-seq data analysis}

We obtained whole-transcriptome sequencing data for G. hirsutum from the NCBI Sequence Read Archive (SRA) (http://www.ncbi.nlm.nih.gov/sra) to analyze the tissue/organ-specific, stage-specific and stress-induced expression patterns of cotton $S O D$ genes. The details of the SRA are shown in Additional file 3. We then used fastq-dump from SRAToolkit.2.4.5-2-centos_linux64 to convert the SRA data into the fastq format (http:// www.ncbi.nlm.nih.gov/Traces/sra). The HISAT2 pipeline was used to build indices and align the clean reads generated from the above steps to their reference genomes (https://ccb.jhu.edu/software/hisat2/index.shtml) [45]. Then, the text file in the SAM format, which was produced by HISAT2, was sorted and converted to the BAM format using the SAMtools program (http://samtools. sourceforge.net/) [46]. StringTie version 1.2.4 (https:// ccb.jhu.edu/software/stringtie/index.shtml) [47] was used to calculate the expression level of each transcript using the reference annotation file of upland cotton in the GFF3 format, which was fragments per kilobase per million reads values. Finally, these values of the GhSOD candidates were extracted and plotted using MySQL and the $\mathrm{R}$ programming language, respectively.

\section{Plant materials and stress treatments}

Upland cotton TM-1 (provided by State Key Laboratory of Crop Biology, Shandong Agricultural University) was employed in this study. The seeds were germinated in a soil mix [peat moss: perlite, $2: 1(\mathrm{v} / \mathrm{v})]$ in plastic pots at $28^{\circ} \mathrm{C}$ in the dark. And identical seedlings were placed in plant growth chambers at temperature regime of $28 / 21^{\circ} \mathrm{C}$, light intensity of 3,300 lx and photoperiod of $16 \mathrm{~h}$ light/ $8 \mathrm{~h}$ dark. And upland cotton TM-1 plants were also grown under standard field conditions (naturally rain-fed with a daytime high temperature of $30{ }^{\circ} \mathrm{C}-37{ }^{\circ} \mathrm{C}$ and nighttime low temperature of $15{ }^{\circ} \mathrm{C}-30{ }^{\circ} \mathrm{C}$ ) in Tai'an, the experimental station of Shandong Agricultural University. For the tissue/ 
organ-specific expression profiling analysis, cotyledons, hypocotyls and roots were harvested from 10-day-old seedlings. Young leaves (5-cm diameter), fully expanded leaves (15-cm diameter) and flowers were harvested from fieldgrown plants. All of the tissues/organs were frozen in liquid nitrogen and stored at $-80{ }^{\circ} \mathrm{C}$ until total RNA was extracted.

For the gene differential expression profiling analysis, the plantlets were subjected to different abiotic stress treatments after the expansion of the first true leaf. The plantlets were cultivated in Hoagland's solution supplemented with $200 \mathrm{mM} \mathrm{NaCl}$ for the salt treatment, $20 \%$ $(\mathrm{v} / \mathrm{v})$ polyethylene glycol 6000 for the drought treatment, and sprayed with $100 \mu \mathrm{M}$ ABA in $0.02 \%(\mathrm{v} / \mathrm{v})$ Tween 20 for the ABA treatment. The leaves of treated plantlets were harvested at 0,12 and $24 \mathrm{~h}$. All of the treatments occurred at $28{ }^{\circ} \mathrm{C}$ with 3,300 lx continuous light, except the cold stress, which was conducted at $4{ }^{\circ} \mathrm{C}$ in a plant growth chamber with $400 \mathrm{~lx}$ continuous light, and the heat stress, which was conducted at $38{ }^{\circ} \mathrm{C}$ in a plant growth chamber with 3,300 lx continuous light. All of the harvested samples were frozen in liquid nitrogen and stored at $-80^{\circ} \mathrm{C}$ until total RNA was extracted.

\section{Real-time reverse transcription-PCR and data analysis}

Total RNA from these samples was isolated using RNAprep Pure Plant Kit (Polysaccharides \& Polyphenolics-rich, DP441) (TIANGEN, Beijing, China). The concentrations of the isolated RNA samples were determined by $1.5 \%$ agarose gel electrophoresis and a NanoDrop 2000 Spectrophotometer (Thermo Fisher Scientific, Wilmington, DE, USA). Reverse transcription PCR was carried out using PrimeScript ${ }^{\mathrm{Tu}}$ RT reagent Kit with gDNA Eraser (RR047A) (TaKaRa, Dalian, China). Transcript levels were determined using a QuantStudio ${ }^{\text {Tm }} 12 \mathrm{~K}$ Flex Real-Time PCR System (Applied Biosystems ${ }^{\mathrm{Tm}}$, Carlsbad, CA, USA) and SYBR Premix Ex Taq ${ }^{\text {Tm }}$ (RR420A) (TaKaRa), with three biological replicates and technical replicates. PCRs included an initial denaturation at $95{ }^{\circ} \mathrm{C}$ for $3 \mathrm{~min}$, followed by 40 cycles at $95{ }^{\circ} \mathrm{C}$ for $10 \mathrm{~s}, 60{ }^{\circ} \mathrm{C}$ for $20 \mathrm{~s}$, and $72{ }^{\circ} \mathrm{C}$ for $30 \mathrm{~s}$ in a reaction volume of $20 \mu \mathrm{L}$ in a 96-well plate. Following the PCR, a melting curve analysis was performed. Cycle threshold was used for the relative quantification of the input target number. Relative fold difference represents the number of treated target gene transcript copies relative to the number of untreated gene transcript copies, and was calculated according to the $2^{-\Delta \Delta C T}$ method [48]; The data were analyzed with Microsoft Office Excel. To normalize the variance among samples, GhUBQ7 (NCBI accession: DQ116441) was used as an endogenous control. Gene-specific primers used for qPCR are listed in Additional file 4 and were designed using Primer Premier 5.0 [49].

\section{Results}

Identification of SOD genes in the G. hirsutum genome The published genome of G. hirsutum (AD1) acc. 'TM-1' made it possible to identify all of the upland cotton $S O D$ genes. The BLAST algorithm was used to search the upland cotton genome with the known $A$. thaliana SOD protein sequences as bait. A total of 18 putative GhSOD genes were identified, and the gene names, sequence IDs and genomic positions were shown in Table 1. Using the same method, $6 \mathrm{CrSODs}$ from the green alga Chlamydomonas reinhardtii (v5.5), six PpSODs from a species of moss, Physcomitrella patens (v3.3), six AmtSODs from Amborella trichopoda (v1.0) and 11 PtSODs from western poplar, Populus trichocarpa, (v3.0) were identified. The upland cotton $S O D$ gene family was expanded compared with in other plant species. The number of SODs was 6 each in Brachypodium distachyon and Hordeum vulgare, 7 each in Oryza sativa, Sorghum bicolor and Setaria italica, 10 in Zea mays, 12 in Musa acuminate, 18 in Triticum aestivum, 8 in A. thaliana, 9 each in G. arboretum and G. raimondii, and 13 in Glycine max (Additional file 1) [6, 11, 12].

The identified upland cotton $S O D$ gene family members encoded predicted proteins, and a physico-chemical analysis showed that the lengths, molecular weights, isoelectric points and instability indices of SOD proteins were within the ranges of 152-467 amino acids, 15.11$49.64 \mathrm{kDa}, 4.84-8.50$ and 2.99-45.13, respectively (Table 1). The $\mathrm{Cu} / \mathrm{Zn}-\mathrm{SODs}$ and Fe-SODs of G. hirsutum were acidic, and the Mn-SODs were basic. The results were similar to the findings in O. sativa [50] and $S$. bicolor [11]. The instability index is a protein measurement that is used to determine whether the protein will be stable in a test tube $(\leq 40$, probably stable; $>40$, probably not stable) [51]. Other than GhCSD7, GhMSD1, GhMSD2 and GhFSD2, most predicted GhSOD proteins were predicted to be stable (Table 1). The results were in accordance with the research on the single $\mathrm{Cu} / \mathrm{Zn}$ SOD in G. hirsutum [52] and Brassica campestris [53].

$\mathrm{Cu} / \mathrm{Zn}$-SODs in G. hirsutum were predicted to localize in the cytoplasm and chloroplasts, Mn-SODs in mitochondria and Fe-SODs in chloroplasts (Table 1). The information in the literature indicated that $\mathrm{Cu} / \mathrm{Zn}$-SODs localize in the cytoplasm, chloroplasts and peroxisomes, and Fe-SODs mainly localize in the chloroplasts, and to some extent in peroxisomes and apoplast, while $\mathrm{Mn}$ SODs localize in the mitochondria [54]. This corroborated our findings. Nevertheless, all of the SOD isoforms $(\mathrm{Cu} / \mathrm{Zn}$-SOD, Mn-SOD, Fe-SOD) were nuclearly coded and, where necessary, were transported to their organellar locations by means of $\mathrm{NH}_{2}$-terminal targeting sequences [55].

The candidate protein sequences were analyzed, using the Pfam database, for the presence of a SOD domain 


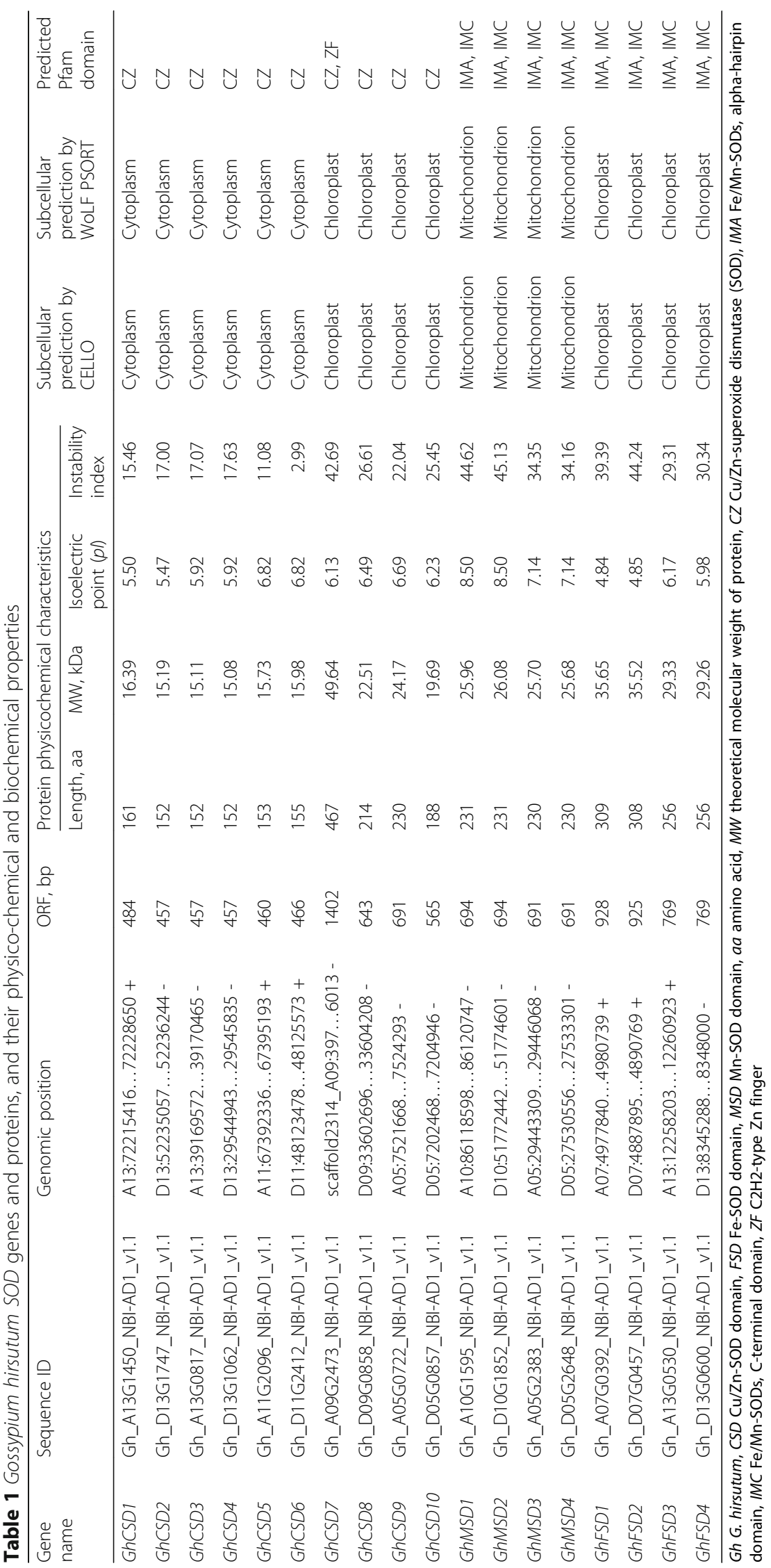


(Table 1). Based on the domain analysis, $\mathrm{Cu} / \mathrm{Zn}$-SODs had a $\mathrm{Cu} / \mathrm{Zn}$ SOD domain (Pfam: 00080) and Mn- and Fe-SODs both had an Fe/Mn-SOD alpha-hairpin domain (Pfam: 00081) and an Fe/Mn SOD C-terminal domain (Pfam: 02777). In addition, similar to our previous findings for G. raimondii SOD genes (GrCSD4) [12], a C2H2-type $\mathrm{Zn}$ finger domain (Pfam: 13912) was found downstream of GhCSD7. Thus, GhCSD7 and GhCSD8 had similar gene structures and close evolutionary relationships, but differed in open reading frame (ORF) lengths, protein lengths, molecular weights and isoelectric points. We aligned the genome sequence and mRNA sequence of GhCSD7 (Gh_A09G2473) with GhCSD8 (Gh_D09G0858) and 5,000 base pair (bp) downstream (D09:33604208.. 33609208) (Additional file 5), and the result preliminarily indicated that gene annotation errors when sequencing the upland cotton genome were the most likely cause.

The MEME server was used for a conserved motif analysis, which identified six conserved motifs. Motifs 1 , 2 and 3 were found to be in $\mathrm{Cu} / \mathrm{Zn}$-SODs, while Motifs 4, 5 and 9 were observed in Mn-SODs and Fe-SODs. Pfam analyses revealed that Motifs 1, 2 and 3 were related to the $\mathrm{Cu} / \mathrm{Zn}$ SOD domain (Pfam: 00080), which contains $\mathrm{Cu} / \mathrm{Zn}-\mathrm{SOD}$ signatures and conserved $\mathrm{Cu}^{2+}$ and $\mathrm{Zn}^{2+}$-binding sites (Additional file 6). Motifs 4, 5 and 6 were related to the Fe/Mn SOD domain (Pfam: 00081, Pfam: 02777), and Motif 6 included the conserved metal-binding domain "DVWEHAYY" of the $\mathrm{Mn}$ - and Fe-SODs. The sequences, locations and logos of the conserved motifs in the GhSOD proteins were shown in Additional files 6 and 7. The data from the Pfam analyses supported our results. Congruent with previous studies in other plant species, the upland cotton $S O D$ gene family contained characteristic amino acids, including a series of highly conserved active site residues that play roles in the sequence-specific binding of mental ions.

\section{Phylogenetic analysis}

To investigate the evolutionary relationships of SODs between Gossypium and A. thaliana, we aligned multiple SOD protein sequences and constructed an unrooted phylogenetic tree for the identified multiple SOD genes using ClustalW and MEGA6.0, respectively (Fig. 1). The GhSOD genes were clustered into two major groups, $\mathrm{Cu} / \mathrm{Zn}$ - and $\mathrm{Mn} / \mathrm{Fe}-$, which showed good accordance with their metal cofactor types. Group I contained three subgroups, Ia, Ib and Ic, represented by brown, purple and yellow, respectively; Group II contained two subgroups, IIa and IIb, represented by green and blue, respectively. According to the subcellular predictions (Table 1), the clustering of group Ia, Ib and Ic may be associated with the subcellular locations of the $\mathrm{Cu} / \mathrm{Zn}$ -
SODs. In the $\mathrm{Cu} / \mathrm{Zn}-\mathrm{SOD}$, those localized to the chloroplasts clustered together with a high bootstrap value (92\%), whereas the cytosolic $\mathrm{Cu} / \mathrm{Zn}-\mathrm{SODs}$ clustered together with a lower bootstrap value (69 and 82\%). Previous research reported different structural gene characteristics between plant $\mathrm{Cu} / \mathrm{Zn}$-SODs observed in the cytoplasm, chloroplasts and peroxisomes, and those in the cytosol and chloroplasts [56]. This supported our results, which showed the separation of chloroplastic and cytosolic $\mathrm{Cu} / \mathrm{Zn}$-SODs. The separation may be affected by the $S O D$ genes' structures, including distinctive numbers and positions of exons (Fig. 2). Additionally, 4 Gh $M n$-SODs and 4 GhFnSODs formed groups IIa and IIb, respectively. This was consistent with our previous results for GaSODs and GrSODs in G. arboretum and G. raimondii, respectively [12].

To further confirm that there were two major SOD groups and to study the evolutionary relationships of the GhSODs and SODs of other plants, we selected a dataset of 70 SOD sequences from 9 flagship species, including 6 from the alga $C$. reinhardtii, 6 from the moss $P$. patens, 6 from A. trichopoda, 6 from $B$. distachyon, 7 from $S$. bicolor, 7 from O. sativa, 8 from A. thaliana, 13 from $G$. max and 11 from $P$. trichocarpa (Additional file 8) that were used as model organisms for studies on plant evolution, and constructed a phylogenetic tree based on their encoded amino acid sequences (Additional file 9). As shown in Additional file 2, the two major groups of the 88 SODs were well supported in this phylogenetic tree. All of the $\mathrm{Cu} / \mathrm{Zn}$-SODs formed a large clade comprising three subgroups (Groups Ia, Ib and Ic). Mn-SODs were clustered with Fe-SODs into another large clade, indicating, as previously reported [6], that these two subgroups (Groups IIa and IIb) originated from a common ancestor. The Chlamydomonas reinhardtii SODs clustered in an independent clade and had no $\mathrm{Cu} / \mathrm{Zn}-\mathrm{SOD}$ members. Additionally, most GhSODs showed closer relationships to SOD proteins from dicotyledonous plants than to those from monocotyledonous plants. These results indicated that land plants may be highly conserved and derived from a common ancestor that may have diverged prior to the split between bryophytes and vascular plants. It also suggested that $\mathrm{Mn}$ - and Fe-SODs were older than $\mathrm{Cu} / \mathrm{Zn}$-SODs, which evolved separately in bryophytes. Our results corroborated the findings of Smith and Doolittle [57].

\section{Gene structure analysis}

To obtain further insights into the possible structural evolution of $S O D$ genes in the upland cotton genome, diverse exon-intron structures of these $S O D$ genes were generated by the GSDS server as shown in Fig. 2b. A gene structural analysis revealed that the ORF lengths of the SOD genes in G. hirsutum ranged from 457 to 


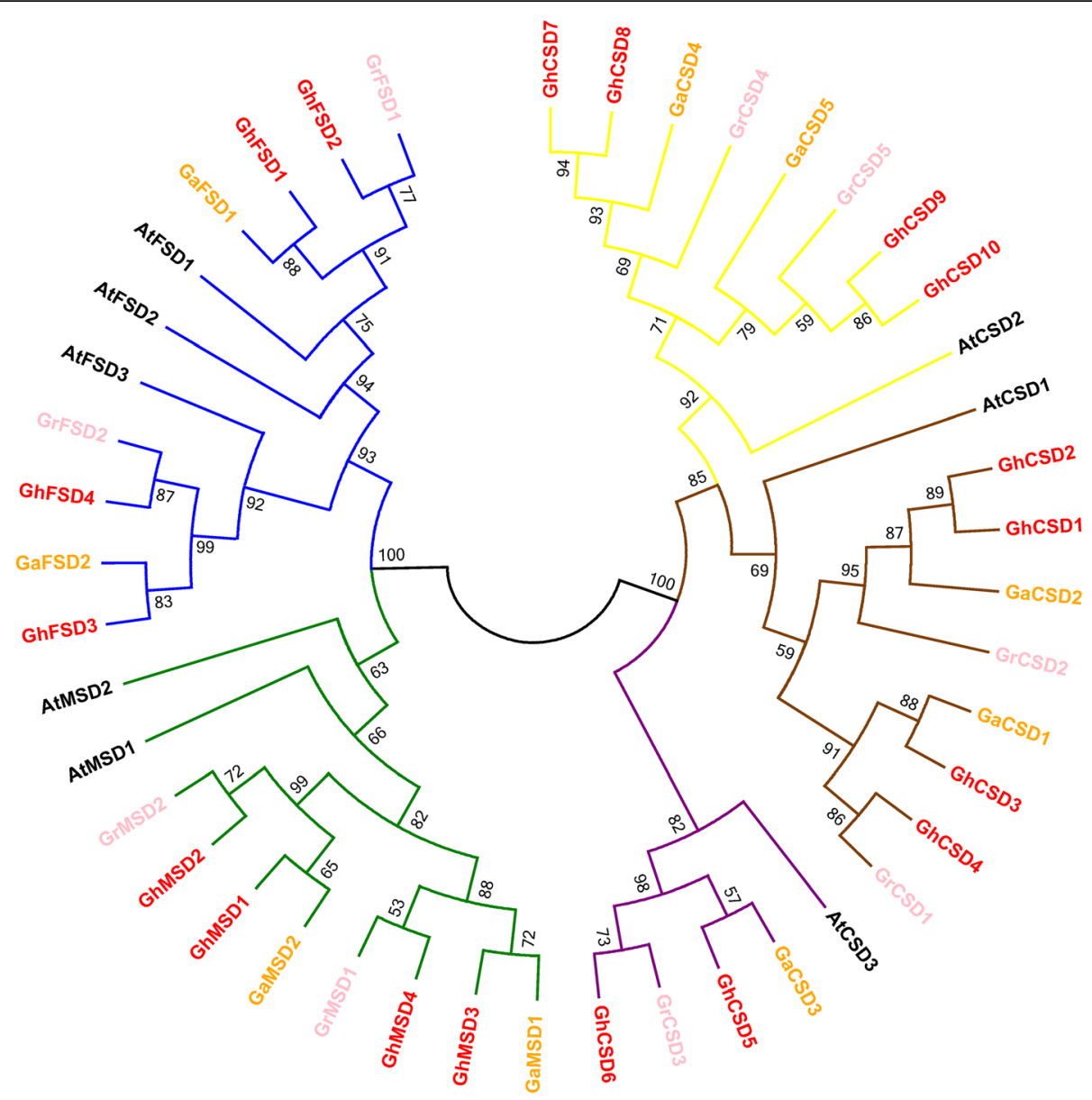

Fig. 1 Phylogenetic tree of SOD genes from the genomes of three cotton species and Arabidopsis. A maximum-likelihood analysis was performed using the MEGA6.0 program. The Jones-Taylor-Thornton with Gamma Distributed substitution model was chosen as the most suitable substitution model based on the result of ModelGenerator before the phylogenetic tree's construction. Red, pink, orange and black gene labels indicate $G$. hirsutum, G. raimondii, G. arboretum and A. thaliana SOD genes, respectively. Bootstrap values obtained from 1,000 replicates are shown below the nodes. The SOD genes were classified into two major groups and five subfamilies (Groups la, lb, lc, lla and Ilb, which have brown, purple, yellow, green and blue branches, respectively)

$1,402 \mathrm{bp}$ (Table 1), and the numbers and positions of introns varied between four and eight (Fig. 2b), with the highest numbers of introns in GhFSD1 and GhFSD2, and the lowest numbers in GhCSD3 and GhCSD4. Fink and Scandalios [56] reported that all of the cytosolic and chloroplastic SODs contained seven introns, except one that had eight introns. The extra intron in the chloroplastic SODs corresponded in location to the second exon of the cytosolic genes. These results were not similar to our findings. In our analysis, only GhCSD7 had seven introns among the 10 chloroplastic and cytosolic $S O D$ s, and the location did not correspond to the second exon of the cytosolic genes. $\mathrm{Cu} / \mathrm{Zn}$-SODs containing different intron numbers and positions showed no exonintron structural similarities in related species [56], and the $\mathrm{Cu} / \mathrm{Zn}$-SODs of G. hirsutum had various intron patterns (Fig. 2b). Thus, our findings were consistent with the data from previous studies. Divergences in exon- intron structures are shaped by three main mechanisms: exon/intron gain/loss, exonization/pseudoexonization and insertion/deletion [58]. Comparing the gene structure of GhCSD1/GhCSD2 and GhCSD7/GhCSD8, the exon/intron gain/loss may occur in $S O D$ genes during the evolution of the G. hirsutum genome, resulting in different intron patterns in G. hirsutum. Furthermore, the sizes and numbers of introns in genes varied depending on the gene and organism types, and it may be related to the functional constraint on the introns [27]. Thus, the differences in intron patterns in G. hirsutum could be explained by functional constraints on the introns of $S O D$ genes [2]. These structural divergences may be related to an enzyme function that responds to various biotic and abiotic stress conditions with expression pattern divergences.

In addition, Mn- and Fe-SODs showed high similarities in sequences and gene structures, especially the 

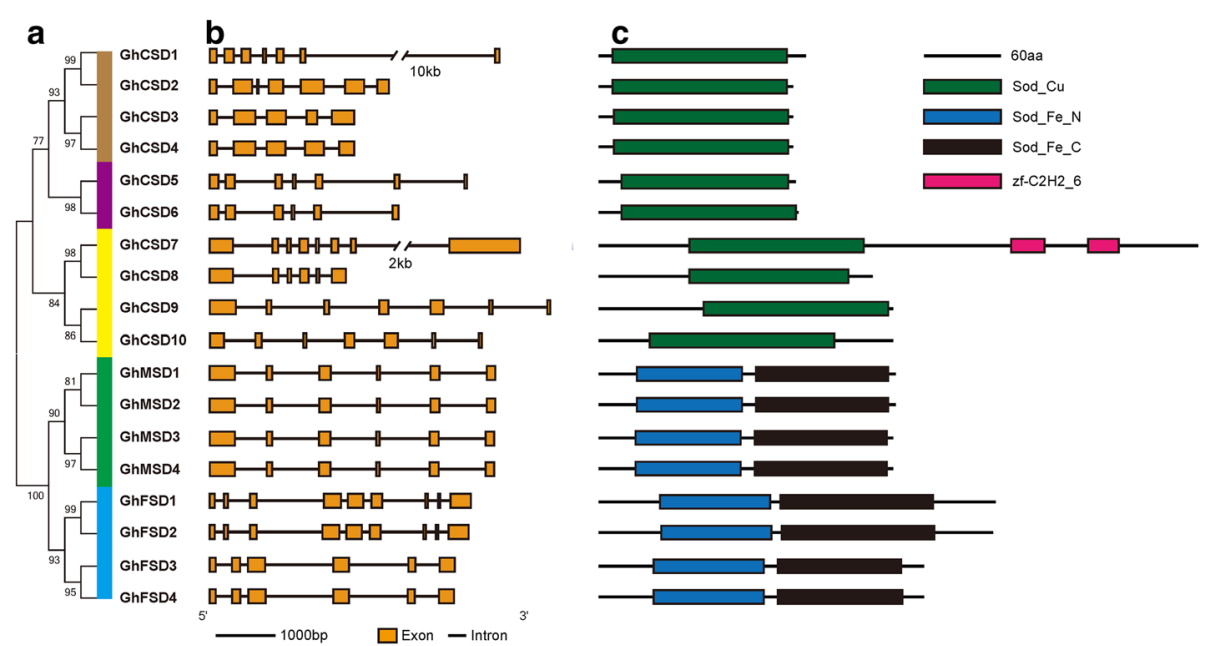

Fig. 2 Phylogenetic tree, and gene structure and domain analyses of superoxide dismutase (SOD) in Gossypium hirsutum. a Phylogenetic tree of G. hirsutum SODs constructed with MEGA 6.0 by the maximum likelihood method. Bootstrap values from 1,000 replicates are indicated at each branch. Groups la, Ib, Ic, lla and Ilb are brown, purple, yellow, green and blue, respectively. b Exon-intron structures of SOD genes. c Conserved domains annotated using the Pfam database

former (Fig. 2b). Mn-SOD was the only SOD form essential for the survival of aerobic life and plants, compared with Fe-SODs and $\mathrm{Cu} / \mathrm{Zn}$-SODs. The results supported Hirsh's hypothesis that the more important the gene/protein, the more conserved [59].

\section{Chromosomal locations and syntenic analysis}

The chromosomal locations of GhSOD genes were determined, and then a chromosomal location map was constructed (Fig. 3). Twelve out of the 26 chromosomes harbored GhSOD genes, with 8 (chromosomes D7, D9, D10, D11, A7, scaffold2314_A09, A10 and A11) possessing one GhSOD genes and 2 (chromosomes D5 and A5) possessing two GhSOD genes, while the others (chromosomes D13 and A13) contained three. Additionally, half of the $18 S O D$ genes were evenly distributed among the D- and A-sub-genomes.

Gene duplications, including segmental and tandem duplications, are primary driving forces in genomic evolution, and paralogous genes are the products of gene duplication events. Duplicate genes playing roles in stress response, development, signaling and transcriptional regulation tended to be retained and formed gene families that were found in almost every genome [60]. To analyze the relationships between the $S O D$ genes and gene duplications, combined with our prior results, we identified the syntenic blocks of $S O D$ genes among $G$. hirsutum, G. raimondii and G. arboreum (Fig. 3). An intra-genome syntenic analysis revealed that three pairs of paralogous genes (GhCSD1 and GhCSD2, GhCSD7 and GhCSD8, and GhCSD9 and GhCSD10) were segmental duplication events and clustered together in G. hirsutum. They are linked together by lines in Fig. 3 .
Segmental duplications may have played important roles in the expansion of $S O D$ genes in the upland cotton genome. The segmental duplication events may provide support for the finer regulation of SOD activities by functional divergences under various stress conditions, and for the temporal- and spatial-specific expressions of SOD genes [61]. Additionally, tandem duplications were not detected. The cross-genome syntenic analysis indicated, excluding $\operatorname{GrCSD2}$, GrCSD4 and GaCSD5, that another eight GaSOD and seven GrSOD genes had orthologous genes in the genome of G. hirsutum (Fig. 3, Additional file 10), suggesting that the other duplicate $S O D$ genes, and the orthologous genes of GrCSD2, GrCSD4 and GaCSD5, in the upland genome were lost after the Gossypium evolutionary event reuniting divergent cotton $\mathrm{A}$ and $\mathrm{D}$ genomes approximately 1-2 million years ago (Mya) [18]. The syntenic blocks of the $S O D$ gene family between the G. hirsutum genome and the two diploid genomes corroborated the results in the genome sequence of allotetraploid cotton (Fig. 3) [19].

\section{Gene ontology (GO) annotations of SODs}

'Biological processes', 'molecular functions', and 'cellular components' are characteristics of genes or gene products that enable us to understand the diverse molecular functions of proteins [39]. GO annotations of 18 upland cotton $S O D$ genes were predicted by considering the orthology and/or homology of $A$. thaliana $S O D$ genes (Fig. 4, Additional file 11). The 'cellular components' data were not in good agreement with the subcellular predictions of some SODs (Table 1). These results may be related to protein sequence similarities caused by genomic events. According to 'molecular functions', all 


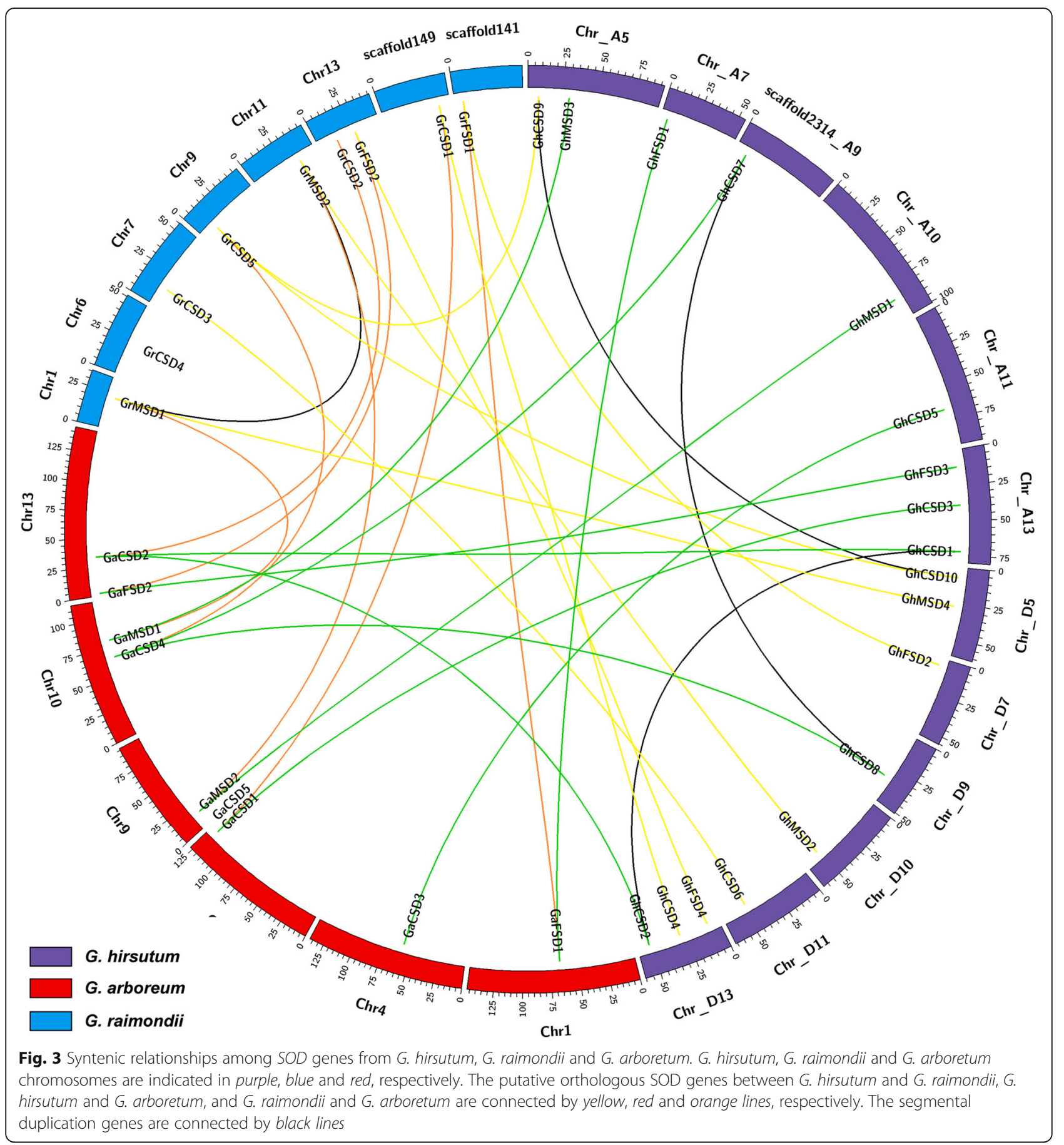

of the GhSOD genes were involved in "superoxide dismutase activity" (GO:0004784), all of the $\mathrm{Cu} / \mathrm{Zn}-\mathrm{SOD}$ genes had "copper ion binding" (GO:0005507) and "zinc ion binding" (GO:0008270), and all of the $\mathrm{Mn} / \mathrm{Fe}-\mathrm{SOD}$ genes had "metal ion binding" (GO:0046872), while the $M n-S O D$ genes belonged to the "copper ion binding" (GO:0005507) group and the $F e-S O D$ genes were in the "protein binding" (GO:0005515) group. 'Biological processes' annotation results indicated that all of the
GhSOD genes contained "removal of superoxide radicals" (GO:0019430) and "oxidation-reduction process" (GO:0055114), except GhFSD1/GhFSD2 only had the latter. Furthermore, the upland cotton $S O D$ genes may be involved in the biological processes responding to biotic stimulus and abiotic stimulus, such as bacterium (GO:0042742), light (GO:0071484), UV-B (GO:0071493), salt (GO:0009651), ozone (GO:0010193), and sucrose metabolism process (GO:0071329). The results corroborated 


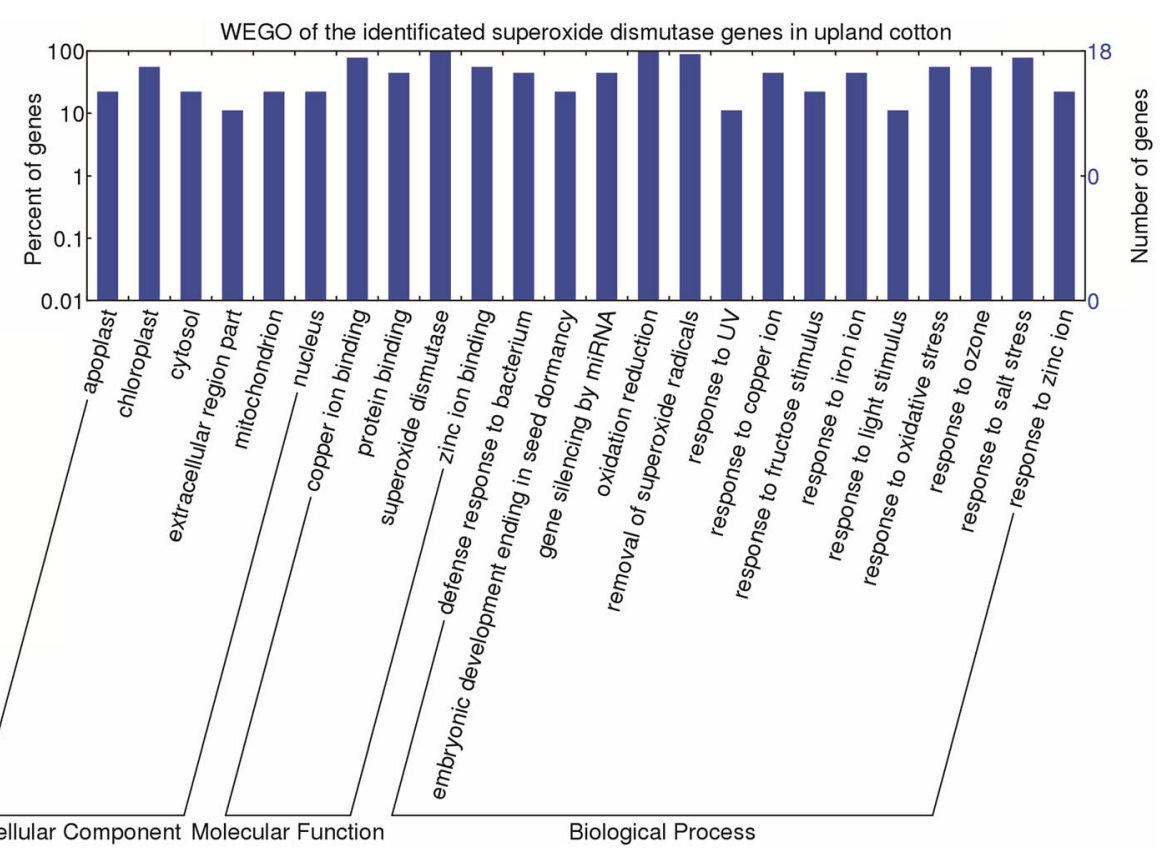

Fig. 4 Functional classifications of the GhSOD genes, according to Gene Ontology Consortium predicted by considering the orthology and/or homology of A. thaliana SOD genes. In this ontology, 'biological process', 'cellular location' and 'molecular function' were treated as independent attributes

the putative GhSOD promoter analysis (Fig. 5). Particularly, the annotation demonstrated that some GhSOD genes' expressions were regulated by microRNA (miRNA) at the posttranscriptional level (GO:0035195). There were some investigations revealed that miR398 targeted and regulated the expression of plant $S O D$ genes in response to biotic stresses, such as salt, drought, heat, high light, ABA, methylation, paraquat and heavy metals [62-64]. The regulation of cotton $S O D$ genes' expression by miRNA needs to be studied further. In addition, GhSOD genes may be involved in reproductive developmental process, such as the

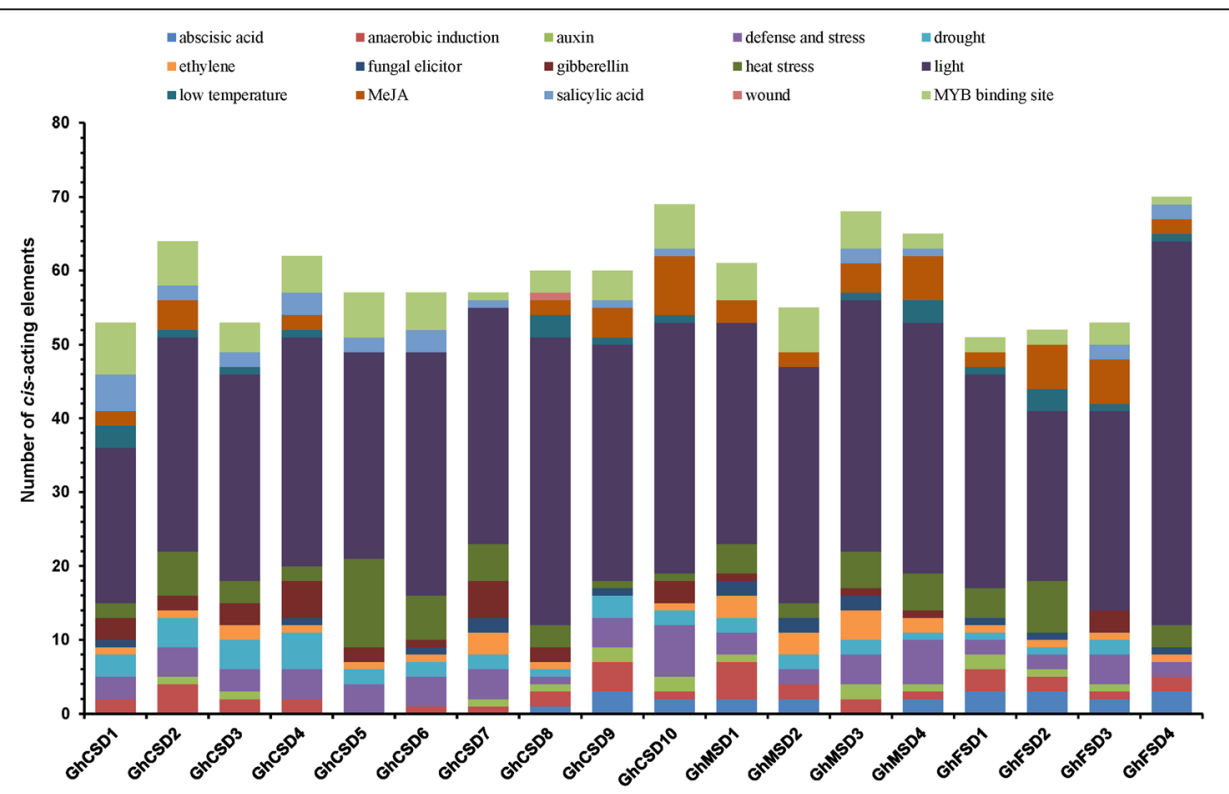

Fig. 5 Cis-element analysis of putative GhSOD promoters related to stress responses. Different cis-elements with the same or similar functions are shown in the same color 
embryonic development ending in seed dormancy (GO:0009793).

\section{Bioinformatics analysis of putative GhSOD promoters}

To further understand and determine the regulatory roles of GhSODs under various stresses, we gathered the GhSOD promoters coupled with 3-kb upstream regions of the ATG start codons from the G. hirsutum genome data downloaded from CottonGen and predicted the transcriptional response elements of the GhSODs' promoters using the PlantCARE tool. All 18 putative GhSOD promoters possessed typical TATA and CAAT boxes, which were the core cis-acting elements in promoter and enhancer regions. Potential regulatory cis-acting elements that were related to stress responses and transcription factor (TF)-binding sites are shown in Fig. 5.

As shown in Fig. 5, 11 kinds of hormone-responsive regulatory elements, ABRE, AuxRR-core, TGA-box, TGA-element, ERE, GARE-motif, TATC-box, P-box, CGTAC-motif, TGACG-motif and TCA-element which were associated with $\mathrm{ABA}$, auxin (IAA), ethylene, gibberellin (GA), methyl jasmonate (MeJA) and salicylic acid (SA) responses, respectively, were found in the GhSOD promoters. And, seven types of stress-responsive regulatory elements, ARE, MBS, Box-W1, HSE, LTR, WUN-motif and TC-rich repeats, with responses to anaerobic induction, drought inducibility, fungal elicitors, heat stress, cold stress, wound stress, and defense and stress, respectively, were identified in the GhSOD promoter regions. In addition, many light-responsive elements existed in all of the GhSOD promoters. There were 29 different types of light-responsive elements present in the 18 upland cotton SOD promoters, and every promoter possessed 4 to 11 types. Increased expression levels of both $\mathrm{Cu} / \mathrm{Zn}$-SOD and Fe-SOD transcripts exist in Arabidopsis, tobacco and rice when exposed to light stress [65]. The constitutive overexpression of the $\mathrm{Cu} / \mathrm{Zn}-\mathrm{SOD}$ gene from pea in tobacco plants conferred a greater resistance to high light levels [66]. Therefore, we proposed that GhSODs may be differentially regulated when subjected to light.

Moreover, all 18 GhSOD promoters possessed myeloblastosis (MYB)-binding sites, including CCAAT-boxes (Fig. 5). MYB-type TFs are widely distributed in all eukaryotic organisms and play important roles in regulating plant biological processes [67]. MYB is widely involved in mediating hormone signaling, cell growth, cell cycle control, primary and secondary metabolism, and cellular morphogenesis in plants [68], and in regulating plant responses to diverse environmental stimuli $[69,70]$. Different types and numbers of regulatory elements were present in the distinct GhSOD promoters, indicating that GhSOD genes should be involved in cotton fiber development and have different regulatory mechanisms in response to various stress and hormone treatments.

\section{Predicting miRNA target sites}

miR398 targeted two of three $\mathrm{Cu} / \mathrm{Zn}$-SODs of Arabidopsis (CSD1 and CSD2) by triggering the cleavage, or inhibiting translation, of their mRNAs [71]. To determine the miRNA-mediated posttranscriptional regulation of GhSODs, we searched the 5' and 3' untranslated regions (UTRs), and the coding regions, of the GhSODs for target sites of $G$. hirsutum miRNAs obtained from various databases and published articles on the psRNATarget server using default parameters. We obtained 20 miRNAs of G. hirsutum that targeted 14 GhSODs at 33 prediction sites. Ghr-miR398 consistently targeted four GhSOD genes (GhCSD7, GhCSD8, GhCSD9 and GhCSD10), and all of the targeted sites located in the coding (CDS) regions. In addition to the $\mathrm{Cu} / \mathrm{Zn}$-SODs targeted by ghr-miR398, other miRNAs of upland cotton targeted GhSODs. GhCSD3 and GhCSD4 were targeted by ghr-miRnF and/or novel_mir_1200 with sites in the second exon; GhCSD7 and GhCSD8 were targeted by m0166 and novel_mir_2733, respectively; GhFSD1 and GhFSD2 were targeted by nine and seven miRNAs with continuously distributed sites, respectively; m0362 and novel_mir_4246 targeted GhFSD3 and GhFSD4, respectively, with sites in the conserved SOD domain; and ghr-miR3 targeted all four $M n-S O D$ genes in upland cotton (Fig. 6, Additional file 2). miRNAmediated posttranscriptional regulation of SODs may possibly be conserved in G. hirsutum. These miRNAs resulted from computational predictions and deep sequencing, and they are involved in some biological processes reported in plants, including responses to environmental stresses [63, 72, 73] and regulating cell growth, development and metabolism in association with cotton fiber development $[74,75]$. The expression profiles of these miRNAs and their targets needed to detect and verify in further experiments to determine their biological functions in upland cotton.

\section{Tissue/organ-specific and stage-specific expression profiles of GhSODs}

A strong link between gene expression and function has been suggested. The SOD gene family is primarily involved in plant growth, development and stress responses. To determine the biological functions in upland cotton, the expression profiles of the 18 GhSOD genes were analyzed in 12 tissues (leaf, petal, seed, cotyledon, torus, root, stamen, pistil, fiber, calycle, stem and ovule) using RNA-seq data recently published by Zhang et al. using the G. hirsutum 'TM-1' plant (Fig. 7) [19]. Not all of the predicted genes in the upland cotton SOD family were expressed in plants grown under normal 

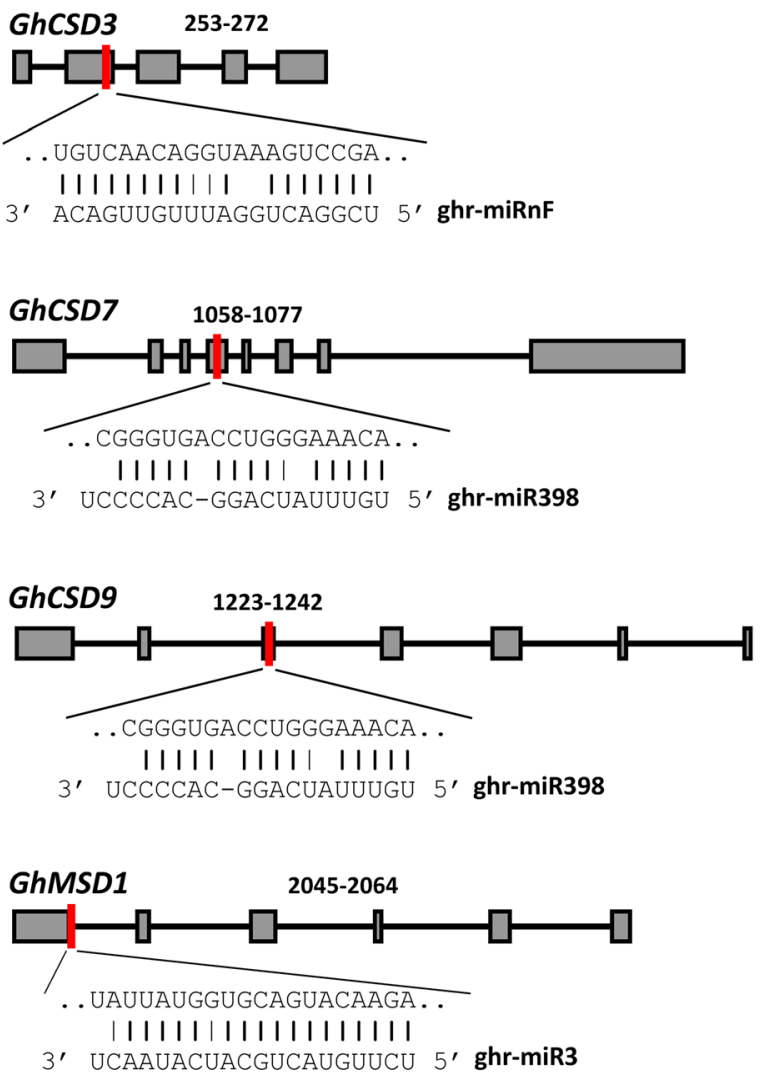

GhMSD3 2655-2674
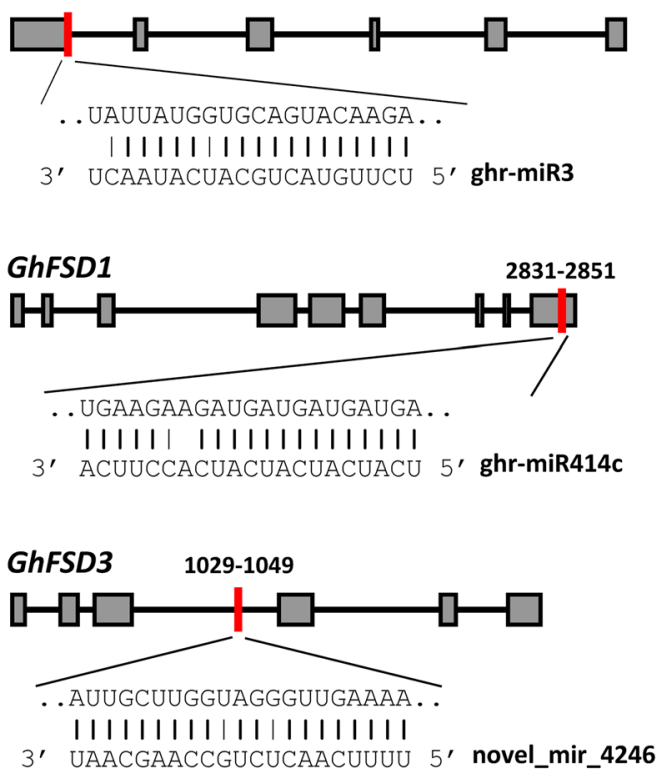
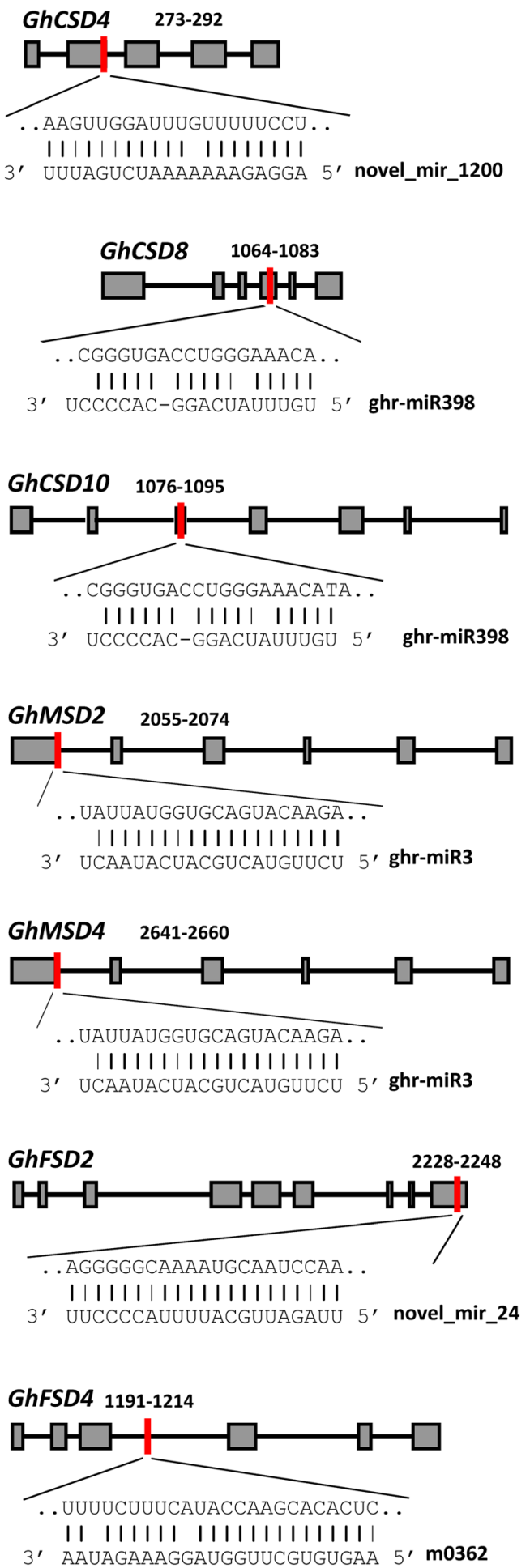

Legend:

$\square$ Exon

- Intron

miRNA complementary sites with the positions of GhSOD gDNAs

Fig. 6 GhSODs targeted by miRNAs of Gossypium hirsutum. The black line represents intron, the rectangle filled grey represents exon, and miRNA complementary sites (red) and the nucleotide positions of GhSOD gDNAs are shown. The RNA sequence of each complementary site from $5^{\prime}$ to $3^{\prime}$ and the predicted miRNA sequence from $3^{\prime}$ to $5^{\prime}$ are shown in the expanded regions 


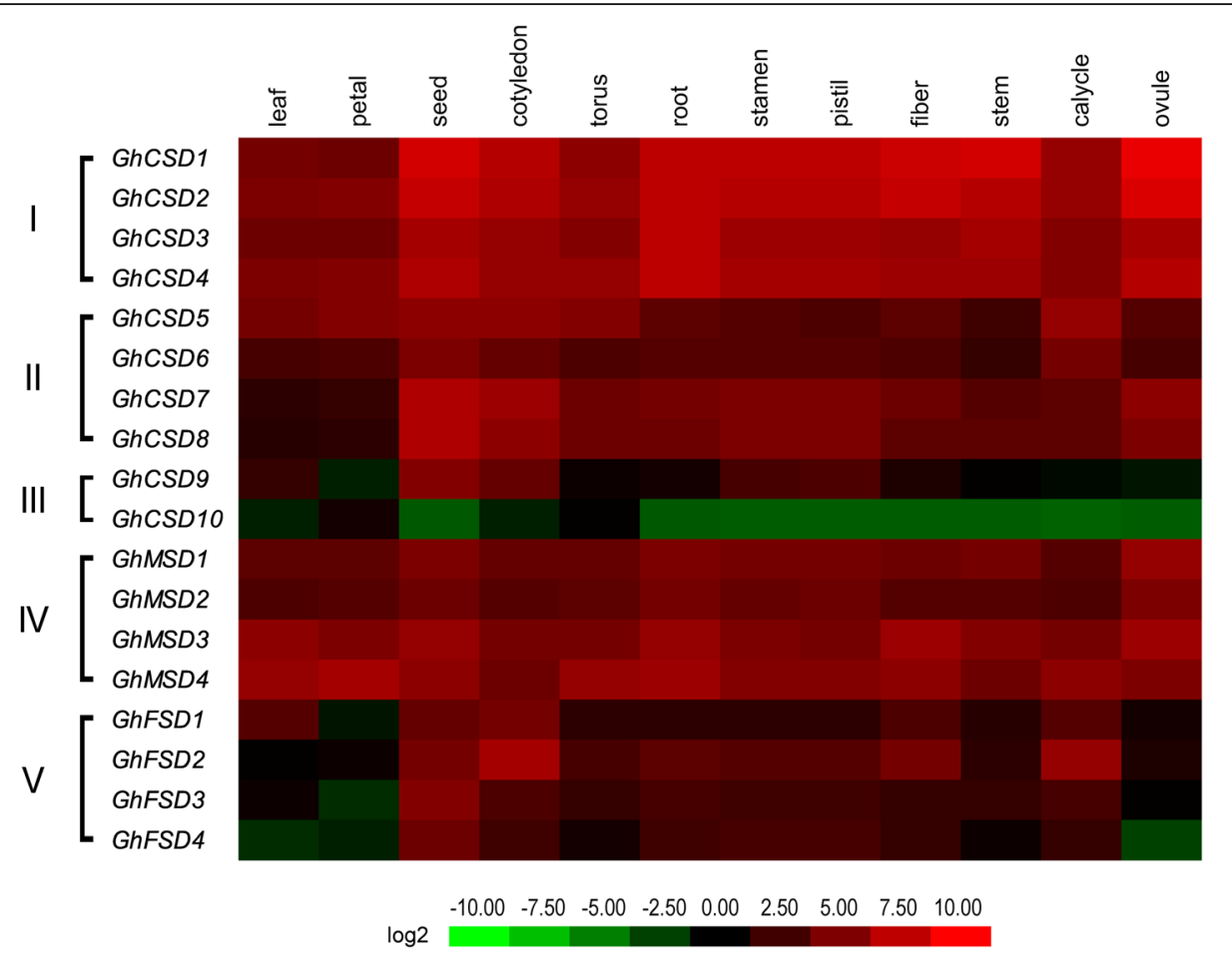

Fig. 7 Tissue/organ-specific expression profiles of GhSODs. Expressions of 18 GhSODs in leaf, petal, seed, cotyledon, torus, root, stamen, pistil, fiber, calycle, stem and ovule were tested. The expression levels of the GhSODs were divided into five groups (I-V). The fragments per kilobase per million reads (FPKMs) calculated by RNA-seq data are shown as a heat map. The colors vary from green to red, representing the scale of the relative expression level

conditions. Among the 18 candidate genes, 17 GhSODs showed detectable expression levels in at least one of the 12 tissues. Additionally, the results enabled the classification of the upland cotton $S O D$ gene family into different groups (I-V) (Fig. 7). The first group was expressed at high levels in almost all of the tested tissues and included GhCSD1, GhCSD2, GhCSD 3 and GhCSD4. The second group was expressed at a slightly lower level compared with the first group and included GhSOD5, GhCSD6, GhCSD7 and GhCSD8. The third group, including GhCSD9 and GhCSD10, had extremely low expression levels, which were almost zero in almost all of the tissues, except GhCSD9 in seed. This suggested that GhCSD10 may be either a pseudogene, or it was expressed at specific developmental stages or under special conditions. The fourth group had higher expression levels than the fifth and second groups and had lower expression levels than the first group, and included GhMSD1, GhMSD2, GhMSD3 and GhMSD4. The fifth group had higher expression levels compared with GhCSD9 and GhCSD10, and included GhFSD1, GhFSD2, GhFSD3 and GhFSD4. Most of the 18 GhSOD genes showed higher expressions in seed and cotyledon but showed lower expressions in leaf, petal and stem, which indicated that the expression of GhSOD genes may be stage-specific or induced under special conditions.
To identify a stage-specific pattern for GhSOD expression, we used the RNA-seq data from seeds, roots, ovules and fibers at different development stages. As shown in Fig. 8, the SOD gene family was expressed at different development stages. Overall, the expression levels of the GhSODs were divided into five groups (I-V), which was consistent with the different tissue/organ tests (Fig. 7). Among the 27 development stages of the 4 tissues/organs, GhCSD1 and GhCSD2 were expressed at a constitutively high level, indicating that these two genes are likely involved in basal metabolic or housekeeping functions in the seed, root, ovule and fiber development of upland cotton, and, remarkably, GhCSD10 was expressed at an extremely low level of almost zero. These results were consistent with the corresponding results shown in Fig. 7. Fiber development has four overlapping stages (initiation, elongation, secondary cell wall biosynthesis and maturation), which were defined on the basis of the number of days post-anthesis [76]. We especially investigated the expression profiles of GhSODs at different fiber development stages. The expression levels of GhCSD1 and GhCSD2 were always high, suggesting they were involved in fiber development. The expression levels of GhCSD3 and GhCSD4 were high during $10-20$ and 35 days post- 


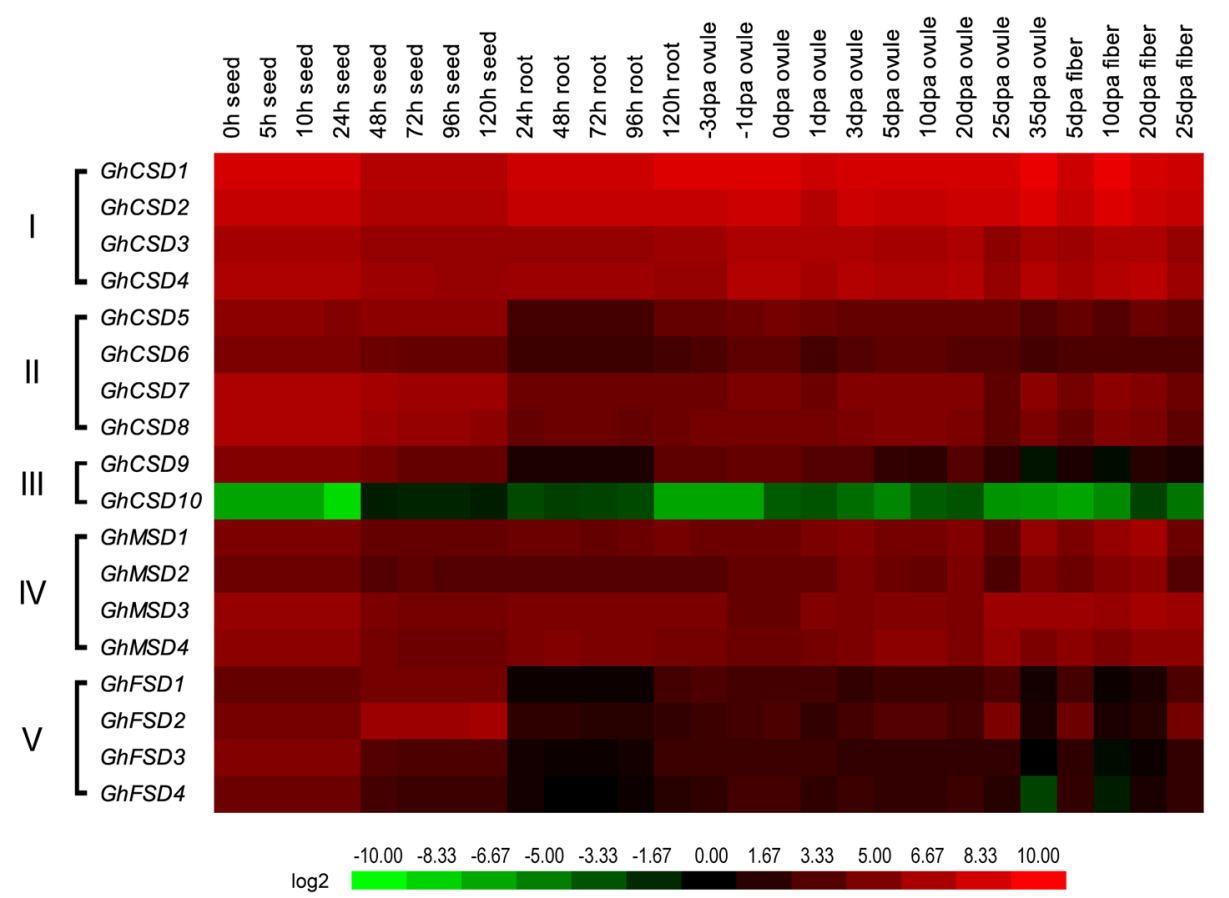

Fig. 8 Stage-specific expression profiles of GhSODs. Expressions of 18 GhSODs in seed, cotyledon, root, ovule and fiber during different developmental stages. The expression levels of the GhSODs were divided into five groups (I-V). The FPKMs calculated by RNA-seq data are shown as a heat map. dpa, day post-anthesis. The colors vary from green to red, representing the scale of the relative expression level

anthesis ovules and fibers, respectively, indicating that they participate in cell elongation, secondary cell wall biosynthesis and fiber maturation. The GhSOD gene family exhibited relatively high expression levels during seed development, except GhCSD10. These results indicated that the $S O D$ gene family plays an important role in the developmental stages of upland cotton fiber.

A qPCR analysis was performed to test and verify the expression patterns of GhSOD genes in different tissues or organs of 'TM-1' (Fig. 9). We used the absolute

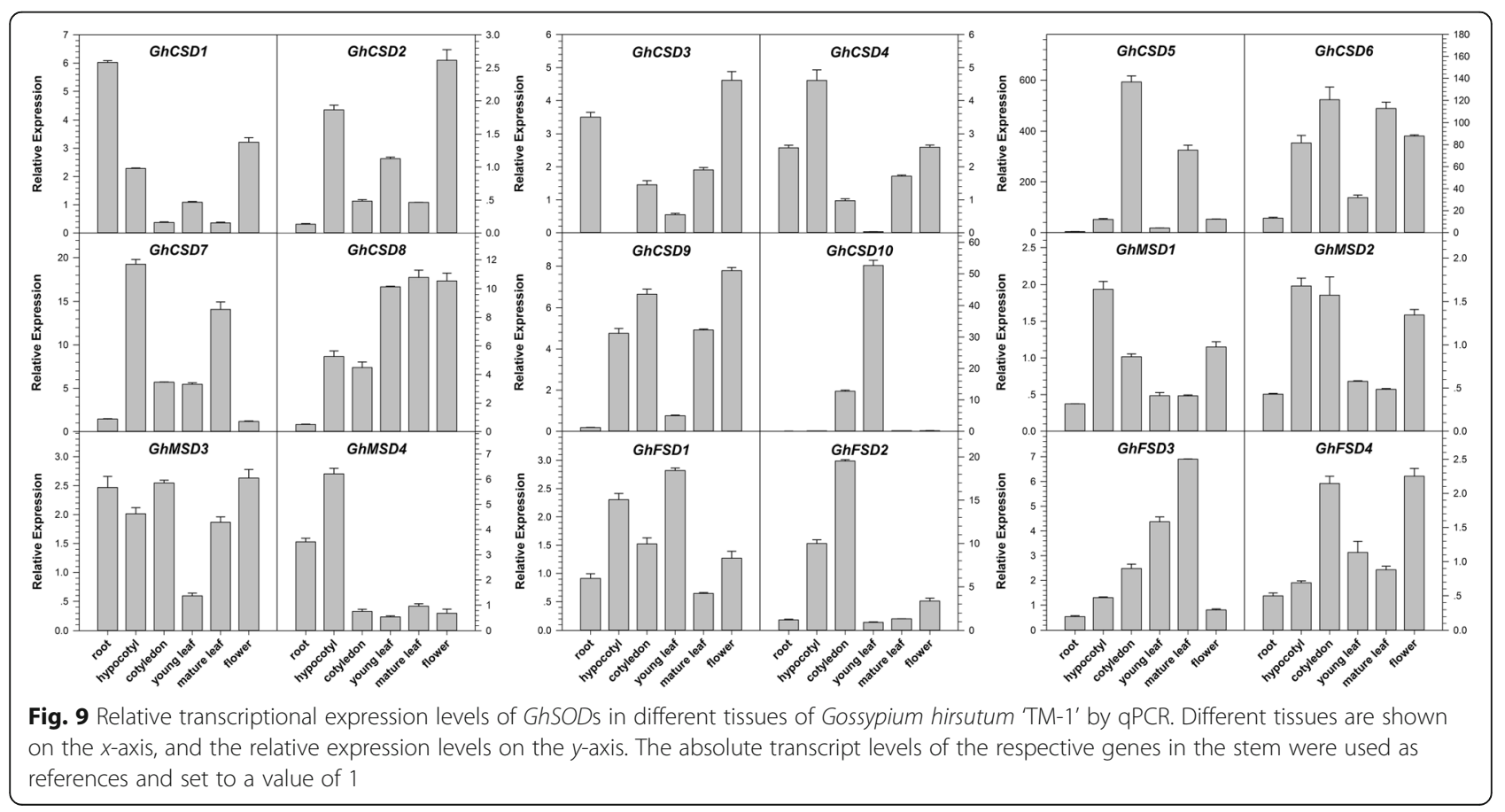


transcript levels of the genes in the stem as references and set the reference value to 1 . According to the results, all of the 18 SOD genes in the upland cotton genome were differentially expressed in all of the tested tissues (root, hypocotyl, cotyledon, young leaf, mature leaf and flower) in non-stressed 'TM-1' plants. Additionally, many GhSOD genes were highly expressed in the cotyledon and mature leaf, followed by in the flower. Cotyledon and mature leaf are the main organs for photosynthesis at the corresponding developmental stages. The photosynthetic electron transport chain, which functions throughout photosynthesis, operates in an aerobic environment. Thus, the high expression level of the upland cotton $S O D$ gene family in cotyledons and mature leaves were related to its active photosynthesis, producing a large amount of ROS. Compared with other tested tissues or organs, GhCSD10 possessed relatively high expression levels in cotyledons and young leaf tissues, but the levels were lower than those of other genes, which corroborated the results shown in Fig. 7. We also detected the different expression levels of homologous $S O D$ gene pairs located on the A- and D-subgenomes of upland cotton. Each homologous GhSOD gene pair showed complementary expression patterns in the same tissue or organ, whereas GhMSD1 and GhMSD2 showed almost the same expression levels. For instance, GhFSD1 was expressed strongly in hypocotyl and young leaf, and weakly in root, while GhFSD2 was expressed weakly in hypocotyl and young leaf, and strongly in root. The complementary expression patterns of the homologous
GhSOD gene pairs indicated that the expression of one gene in the pair was enough to maintain normal physical activity in a non-stressed environment, while the other may be involved in responses to various stresses, together with other genes, or plays important roles in some other growth and development processes. Thus, the $S O D$ gene family members possessing temporal- and spatial-expression specificity may be involved in the growth and development of different tissues or organs of G. hirsutum 'TM-1' plants.

\section{Stress-induced expression profiles of GhSODs}

Cotton is an important economic crop that is widely cultivated in more than 100 countries/regions. Upland cotton contributes $90 \%$ of the yield as the major cultivated cotton species, but its growth, yield and fiber quality are constantly impacted by various abiotic and biotic stresses. Previous studies reported that the plant $S O D$ gene family is widely involved in biotic and abiotic stress responses [3]. High temperatures, extremely low temperatures, drought and salt stresses are the major abiotic stresses that exert detrimental effects on cotton growth and development, causing heavy losses in quantity and fiber quality. To determine the mechanisms involved in the responses of the upland cotton $S O D$ gene family to heat, cold, drought and salt, we detected the expression patterns of all 18 predicted $S O D$ genes in upland cotton under each of the four stresses mentioned above using RNA-seq data (Fig. 10) and qPCR (Fig. 11).

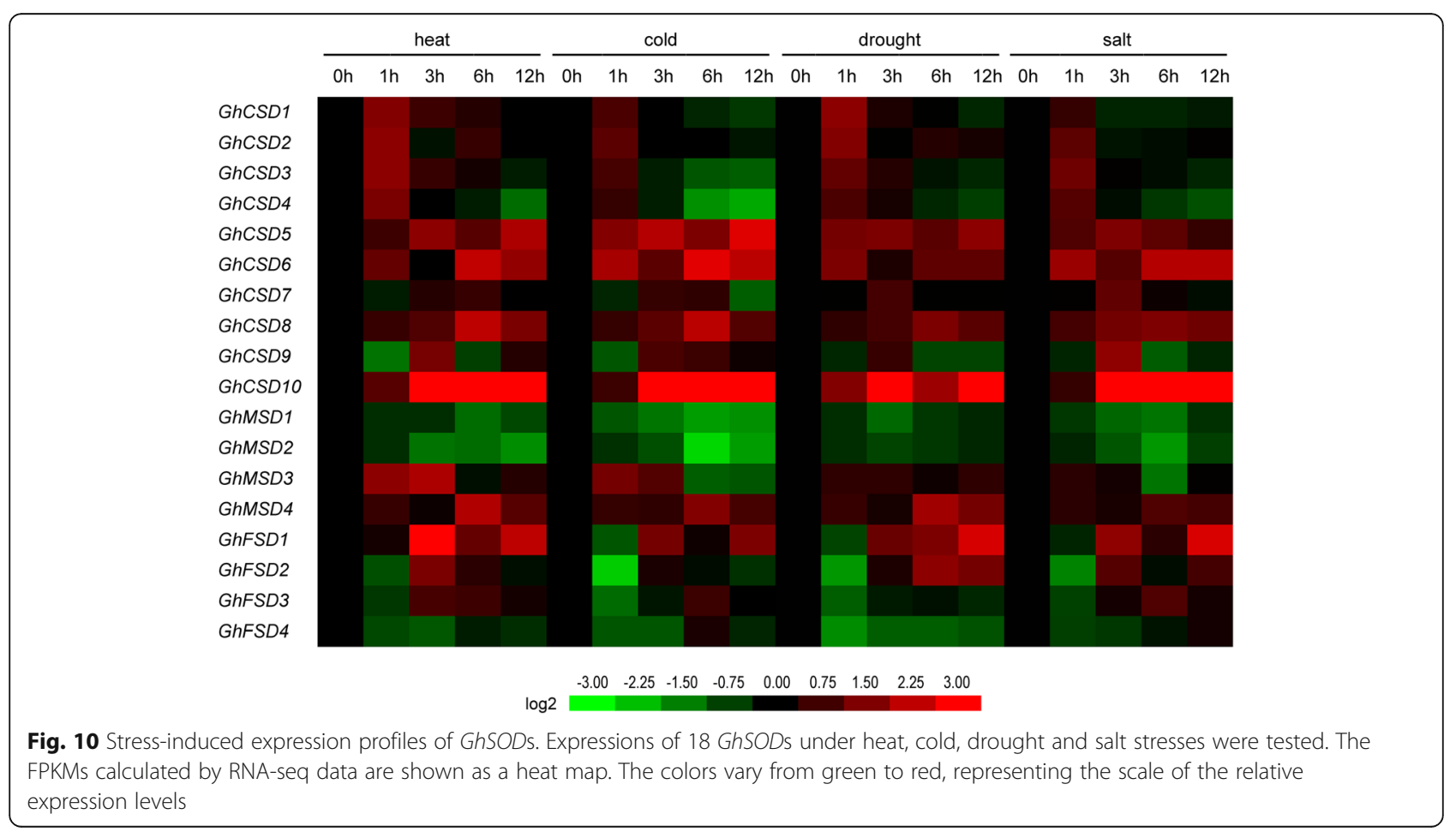




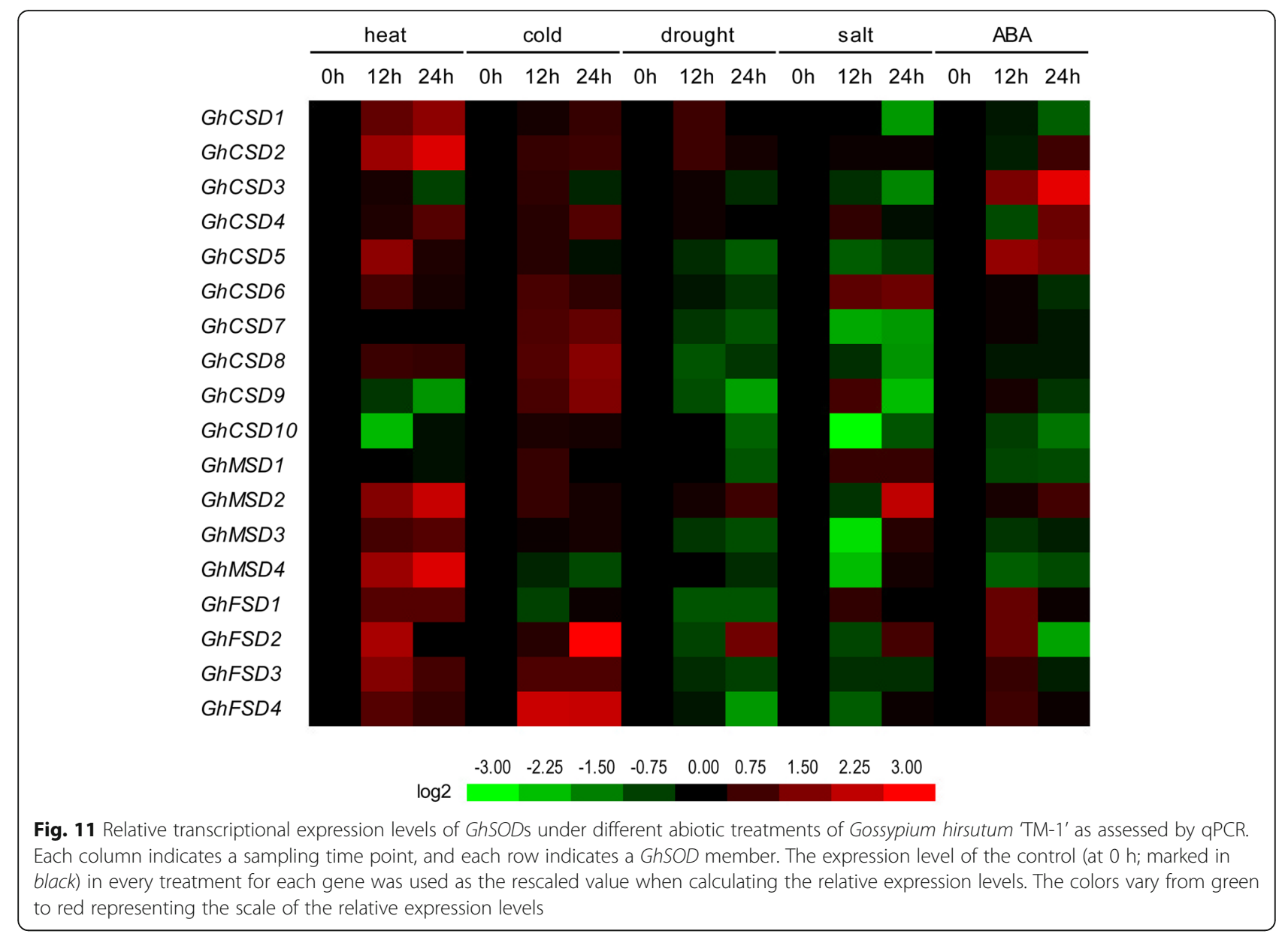

The heat map revealed that the expressions of most $S O D$ genes in upland cotton were induced in the leaves under heat, cold, drought or salt stress conditions (Fig. 10). Under these stress treatments, a total of 5, 5, 4 and 5 genes, respectively, were up-regulated at early treatment time points and down-regulated after experiencing a longer stress treatment. A total of 4, 6, 2 and 2 genes, respectively, were down-regulated at early treatment time points and up-regulated after experiencing a longer stress treatment, indicating the possible existence of a feedback regulatory mechanism. A total of 5, 4 and 1 genes under heat, cold and salt stresses, respectively, were up-regulated throughout the stress treatments, while none of the genes were up-regulated throughout the drought treatment. A total of 3,2, 4 and 3 genes under heat, cold, drought and salt stresses, respectively, were down-regulated throughout these stress treatments. Thus, the genes underlying the comprehensive expression profiles may indicate their vital functions with complex regulatory mechanisms in response to the stress treatments, acting as positive or negative regulators. In addition, there were six (GhCSD5/6/7/8 and GhMSD3/ 4) and three (GhCSD5/8 and GhMSD3) genes that had expression levels that did not clearly change when compared with the control under drought and salt stress conditions, respectively. Thus, GhCSD5/6/7/8 and GhMSD3/4 may not be involved in drought and/or salt stress responses or respond to the two stresses after a long treatment time $(>12 \mathrm{~h})$. Additionally, the relative expression of GhCSD10 was up-regulated continually under heat, cold, drought and salt stresses, but it was also scarcely expressed in the leaf of the control and specific organs (Figs. 7 and 8).

The expression profiles of the 18 GhSOD genes, as assessed by qPCR, were detected independently under heat, cold, drought and salt stresses to validate the varying trends from 0 to $12 \mathrm{~h}$ and provide preliminarily information from 12 to $24 \mathrm{~h}$. The expression patterns were complex (Fig. 11). All 18 SOD genes of upland cotton were heat-treatment responsive. The expression levels of 13 GhSOD genes were up-regulated at $12 \mathrm{~h}$, which corroborated previous results as shown in Fig. 10. And the 13 members (GhCSD1/2/4/5/6/8, GhMSD2/3/4 and the GhFSDs) were continuously up-regulated at $24 \mathrm{~h}$. In contrast, five genes (GhCSD3/7/9/10 and GhMSD1) had reduced expression levels at $12 \mathrm{~h}$ and 
continued to decrease at $24 \mathrm{~h}$. During cold stress, 16 GhSOD genes were up-regulated in response to low temperature stress at $12 \mathrm{~h}$. Among them, 13 genes (GhCSD1/2/4/6/7/8/9/10, GhMSD2/3 and GhFSD2/3/4) were continuously up-regulated at $24 \mathrm{~h}$, except GhCSD3/5 and GhMSD1, which showed no differential expression and were down-regulated at $24 \mathrm{~h}$. The reduced expression of GhMSD4 at $12 \mathrm{~h}$ was increasingly depressed at $24 \mathrm{~h}$, but GhFSD1 showed no differential expression at $24 \mathrm{~h}$. Under the drought treatment, using polyethylene glycol, most of the drought-treatment responsive genes were down-regulated in response to the stress. The transcriptional levels of eight genes (GhCSD5/6/7/8/9, GhMSD3 and GhFSD1/3) were always decreased from 0 to 24 h, except GhFSD2, which was up-regulated at $24 \mathrm{~h}$. Moreover, their expression levels of GhCSD1/2 and GhMSD2 were always increased from 0 to $24 \mathrm{~h}$, and GhCSD4 showed differential expression in response to drought stress. Under salt stress, 12 GhSOD genes were down-regulated at $12 \mathrm{~h}$. Among them, seven genes (GhCSD1/3/5/7/8/10 and GhFSD3) were continuously decreased at $24 \mathrm{~h}$, except GhMSD2/3 and GhFSD2, which were up-regulated at 24 h. GhCSD6 and GhMSD1 exhibited similar expression profiles, which increased gradually to high levels as the $\mathrm{NaCl}$ treatment continued. The expressions of GhCSD4 and GhFSD1 were dynamic, increasing quickly by $12 \mathrm{~h}$, then decreasing gradually to their original levels by $24 \mathrm{~h}$. However, GhMSD4 and GhFSD 4 decreased at $12 \mathrm{~h}$ and increased gradually to their original levels by $24 \mathrm{~h}$. In addition, GhCSD9 was first up-regulated at $12 \mathrm{~h}$, and then down-regulated from $12 \mathrm{~h}$ to $24 \mathrm{~h}$, and GhCSD2 showed differential expression in response to drought stress.

To analyze the potential functions of GhSOD genes involved in phytohormone signaling pathways, we investigated their expression levels in response to ABA (Fig. 11). Three genes (GhCSD3/5 and GhMSD2) were up-regulated to different degrees, whereas another 6 out of the 18 GhSOD genes were down-regulated during the ABA treatment. Among the down-regulated genes, GhCSD1/10 and GhMSD1 showed a continuous highlevel of transcript abundance over the 24 h-time-course, with peaks at $24 \mathrm{~h}$. GhFSDs were strongly induced at $12 \mathrm{~h}$, while GhFSD1/4 and GhFSD2/3 were nonsignificantly induced or down-regulated at $24 \mathrm{~h}$, respectively. Moreover, GhCSD2/4 first showed down-regulation at $12 \mathrm{~h}$, and then were up-regulated from 12 to $24 \mathrm{~h}$. The expression levels of the GhCSD6/7/9 genes exhibited slight variations at $12 \mathrm{~h}$, and then were dramatically down-regulated in response to ABA treatment. The qPCR results corroborated the digital expression profiles of the transcriptome data and suggested that the $S O D$ gene family of upland cotton may be important both for stress responses and developmental processes. Candidate genes from the $S O D$ gene family should be selected for functional analyses of their roles in response to heat, cold, drought and salt stresses.

\section{Discussion}

Recently, with whole-genome sequencing being completed in various plants, studies of related gene families have rapidly progressed. The $S O D$ s are a critically important gene family encoding SOD proteins, which act as the first line in antioxidant systems and play important roles in responding to various environmental stimuli in plants, such as drought, salinity, cold, heat and auxin [3]. The $S O D$ responses to stresses are dramatically different, depending on the different SOD members present, the stress and the plant species. Considering the potential functional significance of the $S O D$ gene family and that several of its members have been identified in G. raimondii and G. arboretum [12], characterizing the $S O D$ gene family in upland cotton is important.

\section{Expanded SOD genes family in G. hirsutum (AD1) genome} In this study, we identified 18 upland cotton $S O D$ genes clustering into two major groups $(\mathrm{Cu} / \mathrm{Zn}-\mathrm{SODs}$ and $\mathrm{Mn} /$ $\mathrm{Fe}$-SODs), having 9 members (5 CSDs, $2 \mathrm{MSDs}$ and 2 $F S D$ s) in the A-subgenome and 9 members (5 CSDs, 2 $M S D$ s and 2 FSDs) in the D-subgenome. The number of $S O D$ genes varies among plants, as shown in Additional file 1 [6]. Excluding the $S O D$ gene family of hexaploid bread wheat, the number of $S O D$ genes in diploid cottons was greater than that in Algae and Bryophyte, was similar to that in Poaceae and Cruciferae, and was less than that in banana, poplar and soybean. Whole-genome duplication (WGD) or polyploidy events occurred throughout the evolutionary history of many flowering plants [77]. For instance, one WGD event occurred at the root of the seed plants 310 Mya, and another paleohexaploidization event at the outset of eudicots 130-190 Mya. Two WGD events occurred in monocots and may pre-date the diversification of Poaceae, and one whole genome triplication (WGT) event was probably shared by all of the core eudicots. Two recent WGDs occurred within the crucifer lineage and other lineagespecific WGD or polyploidy events occurred during the evolutionary progress of some plants, such as maize, bread wheat, banana, diploid cottons, poplar and soybean (Addition file 1) [78, 79]. These common and lineage-specific WGD or polyploidy events in plants, which generated duplicate copies of every gene, are the major factor responsible for the $S O D$ gene family expansion. However, the $S O D$ gene family members are not in exact proportion with the WGD or polyploidy events. Based on the phylogenetic and syntenic analyses of the upland cotton SOD gene family, we 
hypothesize that this was most likely caused by considerable gene loss, resulting from the deletions or insertions of genes, sequence divergence that occurred through point mutations, and the rearrangement or loss of chromosomes, after WGD or polyploidy events during upland cotton's evolution. GhCSD1/GhCSD2, GhCSD7/GhCSD8 and GhCSD9/GhCSD10 clustered together in groups Ia, and Ic, and had first exons of identical size and high sequence similarities (Figs. 1 and 2). Hence, they may be potentially derived from segmental duplications and originated from $\operatorname{GrCSD} 2$, GaCSD4 and GrCSD5, respectively.

By further analyzing the SODs in the G. raimondii genome, we obtained more than one kind of transcript. Specifically, the number of transcripts was two for $\operatorname{GrCSD} 3$, and three each for GrCSD1, GrFSD1 and GrFSD2. An analysis of alternative splicing (AS) suggested that GrCSD1, GrFSD1 and GrFSD2 probably underwent three basic types of alternative RNA splicing events, alternative 3' splice site, intron retention and 5 ' splice site (Additional file 12). Like AS, alternative polyadenylation (APA) and alternative transcription sites (ATSS) are other regulatory mechanisms that form transcript variants from a single gene. APA and ATSS transcripts have been detected in SOD genes from Musa acuminate [6], Dimocarpus longan [4], Larix gmelinii [80], O. sativa [81] and T. aestivum [82]. In this study, because of the absence of access to transcript variants of the upland cotton $S O D$ gene family, we did not continue the analysis. However, various previous reports in plants suggested that $S O D$ variants generated by AS, APA or ATSS were linked to regulating spatial- and temporal-gene expression [83], and may play a crucial role in responding to some abiotic stresses [81]. Thus, a future research project will be the cloning of the fulllength cDNAs of the 'TM-1' $S O D$ gene family, and analyzing and confirming the formation and functional mechanisms of $S O D$ variants using a nextgeneration sequencing technique, such as RNA-seq.

Overall, we hypothesize that WGD or polyploidy events and segmental duplications contributed to the expansion of the upland cotton $S O D$ gene family. Additionally, the complex regulation at the transcriptional level, such as through the AS of the premRNA, generated two or more protein isoforms from single genes, contributing to the SOD protein diversity. The viable variations in SOD caused by AS, facilitated by an increasing abundance of SOD protein disorder in Gossypium would have provided an avenue for natural selection, which may have even facilitated the functional diversification of the upland cotton $S O D$ gene family over relatively short periods on the geological time scale.

\section{SOD gene family evolution and genomes evolution in plants}

During the evolution of Gossypium, many WGD or polyploidization events that caused species differentiation through polyploid formation occurred, including an ancestral seed plant WGD, an ancestral angiosperm WGD and one WGT that was probably shared by all of the core eudicots. Shortly after divergence from cacao, the Gossypium lineage experienced a five- to six-fold ploidy increase and a subsequent diversification into eight diploid genome groups, including $\mathrm{A}-\mathrm{G}$ and $\mathrm{K}$. A-genome diploids native to Africa and Mexican D-genome diploids diverged approximately 5-10 Mya. These two species were reunited geographically approximately 1-2 Mya by the transoceanic dispersal of an A-genome ancestor resembling G. arboreum to the New World. Then, hybridization with a native $\mathrm{D}$-genome species resembling G. raimondii and chromosome doubling occurred, forming the common ancestor of G. hirsutum (Upland) and G. barbadense (Egyptian, Sea Island and Pima) cottons $[18,84]$. Combined with the analyses of phylogenetic and syntenic relationships of $S O D$ gene families from G. hirsutum, G. arboreum and G. raimondii (Figs. 1 and 2), we showed that the overall gene order and the co-linearity of the $S O D$ gene family in upland cotton were largely conserved between the A- and Dsubgenomes and the extant D-progenitor genome ( $G$. raimondii). Thus, we believe that, during the evolution of the Gossypium SOD gene family, one gene was native to the D-genome ancestor and the other one was native to the A-genome ancestor for the alleles of the GhMSDs, GhFSDs, and GhCSD3/GhCSD4 and GhCSD5/GhCSD6. Meanwhile, GhCSD1/GhCSD2, GhCSD7/GhCSD8 and GhCSD9/GhCSD10 separately originated from GaCSD2, GaCSD4 and GrCSD5 and were formed by gene duplication. However, GrCSD2, GrCSD4 and GaCSD5 were lost after the reuniting of the A- and D-genome ancestors, which may be caused by the biased fractionation of the genome [85] and/or subgenome dominance [86]. This is just one of our hypotheses, because G. arboreum and G. raimondii are the only two extant progenitor relatives, and the exact donor species that led to the formation of the tetraploid cotton species 1-2 Mya no longer exists [84].

The $S O D$ genes are an important gene family encoding SOD proteins, which play a major role in the defense system against oxidative stress in plants and are ubiquitous in every cell of all plant types. Several SOD homologs have been discovered in many plant species, including green algae, moss, Amborella, Arabidopsis, rice, Brachypodium, durra, soybean and poplar. Based on the phylogenetic relationships of the $S O D$ gene family from upland cotton and the nine flagship species considered pivotal references for understanding plant 
evolution, the $S O D$ gene family of plants was divided into two major groups $(\mathrm{Cu} / \mathrm{Zn}$ - and $\mathrm{Mn} / \mathrm{Fe}-)$. $\mathrm{Cu} / \mathrm{Zn}$ $S O D$ s contained three subgroups (Ia, Ib and Ic) and $M n /$ $\mathrm{Fe}$-SODs contained two subgroups (IIa and $\mathrm{IIb}$ ) (Additional file 9). This grouping agreed with the results shown in Fig. 1. Additionally, the phylogenetic relationships of $S O D$ genes in each subgroup were consistent with the evolution of plants (Additional files 9). Remarkably, the green algae $C$. reinhardtii had $5 \mathrm{Mn}$-SODs, 1 $\mathrm{Fe}-\mathrm{SOD}$ and no $\mathrm{Cu} / \mathrm{Zn}$-SODs, and the $5 \mathrm{Mn}$-SODs possessed high sequence similarity. This suggested that the $\mathrm{Mn}$ - and $\mathrm{Fe}$-SODs were older than the $\mathrm{Cu} / \mathrm{Zn}$-SODs because they are thought to have been generated from the same ancestral enzyme, while the $\mathrm{Cu} / \mathrm{Zn}$-SODs evolved separately and did not possess such a similarity [57]. Meanwhile, the number of $M n-S O D$ s in green algae was greater than in all of the 18 plants, and the number of $\mathrm{Cu} / \mathrm{Zn}$-SODs was greater than that of the $\mathrm{Mn}$ - and $\mathrm{Fe}$ $S O D$ s in plant evolution (Additional file 1). On the early earth, the primitive levels of oxygen could not exceed that of molecular oxygen $(\sim 0.001 \%)$ present at the atmospheric level, and metal ions, such as $\mathrm{Mn}$ and Fe, were relatively easy to obtain [87]. Accordingly, $\mathrm{Mn} / \mathrm{Fe}-$ SODs were in a dominant position, especially Mn-SODs. With the evolution of earth, the rise of oxygen from the primitive levels could only be associated with oxygenic photosynthesis that was well-established on the earth at least 3.5 thousand Mya, thereby contributing to the concentration of ROS. Furthermore, Mn and Fe ions participated in the electron transport chain as an indispensable part of the two photosystems' reaction centers. Meanwhile, plants were facing a more complicated and uncertain [88]. Therefore, $\mathrm{Cu} / \mathrm{Zn}$-SODs separated from the family and occupied the dominant position during plant evolution to respond to the various abiotic and biotic stresses. Thus, we hypothesize that with the everchanging earth environment and the plant evolution, the $S O D$ gene family expanded and experienced biased fractionation after multiple rounds of polyploidization events in plant genomes, particularly massive $M n-S O D$ gene losses. Moreover, $\mathrm{Cu} / \mathrm{Zn}$-SODs evolved separately in bryophyta with the functional differentiation, caused by including neofunctionalization and/or epigenetic modifications of transposable elements, of $M n-S O D$ s to response to various stresses [89].

Thus, comparative sequence and phylogenetic analyses of the three plant $S O D$ isoforms suggested that $\mathrm{Mn}$ - and $\mathrm{Fe}$ $S O D$ s may have arisen from common ancestral enzymes, whereas $\mathrm{Cu} / \mathrm{Zn}$-SODs evolved separately in bryophyta. The two major groups must have evolved independently.

\section{Gene expression patterns of GhSODs}

The spatiotemporal expression patterns of SODs have been determined in many species, such as Arabidopsis, longan and banana. For banana, 11 of 12 MaSODs were expressed at relatively high levels in the leaf, pseudostem and root [6]. All DlSOD types with moderate and stable expression, such as DlCSDs, DlMSD and DlFSD, mediated development during the four primary developmental stages of longan early somatic embryogenesis [4]. In this study, we analyzed the transcript levels of all of the GhSODs in 12 different organs/tissues and some different development stages. Most of the GhSODs are expressed predominantly in cotyledon, leaf, flower and seed, with relatively weak expressions in the stem, root and fiber. Cotyledon and leaf are major vegetative organs that play fundamental roles in the maintenance of plants life through photosynthesis. Additionally, flower and seed undergo organogenesis, germination and other metabolic processes as common reproductive organs. Large amounts of ROS are often generated during these vital processes and, therefore, the expression levels of the GhSODs were relatively high in these organs/tissues. Our data indicated that GhCSD1 and GhCSD2 were involved in whole-plant development, and that most of the other GhSODs may play important roles in seed, root, ovule and fiber development (Fig. 8). Additionally, the tissue/organ-specific and stage-specific expression profiles of the GhSODs suggested that the expression yields were in accord with their phylogenetic clustering results (Figs. 1 and 2a), namely, that the genes that were grouped in one of the Ia, Ib, Ic, IIa or IIb classes shared similar expression patterns in different tissues/organs and developmental stages. Thus, the $S O D$ genes of upland cotton were expressed with a certain degree of temporal- and spatial-specificity and played important roles in different tissues/organs and developmental stages.

Previous studies reported that plants could maintain the ROS balance by ROS-scavenging systems caused by abiotic stress that mainly involved three types of SODs, $\mathrm{Cu} / \mathrm{Zn}-\mathrm{SOD}, \mathrm{Mn}-\mathrm{SOD}$ and Fe-SOD [90]. The expression analysis suggested that every GhSOD gene responded to at least one abiotic stress performed in this study (heat, cold, drought or salt) (Figs. 10 and 11), which coincided with the GO annotations of the SODs (Fig. 4 and Additional file 11) and bioinformatics analysis of putative GhSOD promoters (Fig. 5 and Additional file 2). The results of the GO annotations revealed that the $S O D$ gene family of upland cotton may be involved in biological processes, including responses to biotic and abiotic stimuli, such as bacterium, light, UV-B, salt, ozone and sucrose metabolic processes. Moreover, according to the analysis of putative GhSOD promoters, the promoters of the GhSOD gene family harbored more kinds and numbers of cis-elements involved in abiotic stresses, including the cis-elements involved in lowtemperature responsiveness, cis-elements involved in heat stress responsiveness, MYB-binding sites involved in 
drought-inducibility and cis-elements involved in the ABA responsiveness, which could explain why GhSOD exhibited obvious responses to the four abiotic stresses. Notably, compared with GhMSDs and GhFSDs, GhCSDs showed obvious expression changes under all four abiotic stresses. This indicated that GhCSDs may play a predominant antioxidant role in upland cotton. Similar observations were also detected in other plant $\mathrm{Cu} / \mathrm{Zn}$ SOD genes [6, 91].

Hormone-responsive TFs regulated the expression of target genes by combining with their corresponding ciselements in the promoters during various stresses. The analysis of putative GhSOD promoters predicted that 10 GhSOD promoters (GhCSD8/9/10, GhMSD1/2/4 and GhFSDs) harbored one to three ABREs, a cis-acting element involved in the $\mathrm{ABA}$ responsiveness, indicating that these genes probably participate in ABA responses. Moreover, the expression of eight other GhSODs, which had no ABREs, showed differential expression inductions during the $A B A$ treatment, suggesting that there were other regulatory mechanisms responding to $\mathrm{ABA}$, such as miRNA. Tang et al. reported that miR398 negatively regulated the expression of its target genes $(\mathrm{Cu} / \mathrm{Zn}$ $S O D s)$ in response to ABA [63]. In this study, we detected that ghr-miR398 targeted GhCSD7, GhCSD8, GhCSD 9 and GhCSD10, and that all of the targeted sites were located in CDS regions, which may explain why some of the GhSODs responded to the ABA treatment. We, therefore, proposed that the GhSODs expression in response to ABA may work in coordination with ABREs and miRNAs. In addition, there were 10 other hormoneresponsive cis-elements (TGA-element, ERE, CGTAmotif, CGTCA-motif, TGACG-motif, GARE-motif, P-box, TATC-box, AuxRR-core and TCA-element) located in the putative promoter regions of the GhSODs. Their transcriptional regulation under IAA, GA3, methyl jasmonate and SA treatments needs to be investigated in further experiments. We hypothesize that the $S O D$ genes of upland cotton probably participate in phytohormone signaling pathways.

\section{Regulation of GhSOD genes expression}

Plants utilized a complex regulatory network of transcriptional, posttranscriptional, translational and posttranslational gene expression programs to respond to various abiotic and biotic stresses. Characterization of TFs and stress-responsive regulatory cis-elements offers insights into the upstream regulation of GhSODs. Cisacting elements involved in stress-induced gene expression were predicted in the promoter regions of the GhSOD genes. Additionally, MYB-type TF-binding sites were obtained in all 18 GhSODs (Fig. 5). The various functions for the MYBs has been investigated in numerous plant species using both genetic and molecular analyses. MYB plays crucial roles in different processes, including primary and secondary metabolisms, such as the regulation of various phytochemical biosynthesis pathways, regulation of several developmental processes, such as cell fate determination in root hairs, secondary cell wall biosynthesis, establishment of the axillary branch patterning, leaf proximodistal axis and anther development, and responses to environmental stimuli [92, 93]. For instance, in upland cotton, GhMYBs may be involved in regulating specialized outgrowths of epidermal cells, including cotton fiber development during different developmental stages [93], and several $M Y B$ genes in cotton were differentially expressed under salt and drought stress treatments [92]. Additionally, many $M Y B$ genes in other plant species were involved in regulating responses to biotic and abiotic stresses, and enhanced the tolerance to stresses in transgenic plants $[94,95]$. GhSODs may also be involved in cell wall growth and development processes, including cotton fiber development [96], and in responding to environment stresses and enhancing the tolerance of transgenic plants expressing $S O D$ genes against oxidative stress [3]. Using bioinformatics analyses of putative GhSOD promoters, we obtained several binding sites of MYB TFs. The expressions of $S O D$ genes may be regulated by MYB-type TFs. Hence, we hypothesized that MYB could control the expression of upland cotton $S O D$ genes in response to environmental stresses and regulate the process of cotton fiber development at the transcriptional level. The functions of most MYBs in higher plants remains unclear, and the roles of MYB in SOD regulatory networks, as well as their inferred functions, remains to be fully elucidated through the use of inducible systems in high-throughput expression and interaction studies, combined with bioinformatics and systems analyses in G. hirsutum.

miRNAs are a diverse category of nuclear-encoded small RNAs that play multiple, central functions in plant development, stress responses, and many other biological processes. Mature miRNAs facilitate the cleavage of bound target genes and/or trigger translational repression by binding to the 5' UTR, 3' UTR or coding regions of the target mRNAs [97]. Currently, miRNA-mediated posttranscriptional gene regulation is particularly interesting because miRNAs can regulate several protein-coding genes implicated in the same pathway.

In our study, we obtained some predicting miRNA target sites locating at introns. For instance, novel_miR_47/ $50 / 51$, identified in G. hirsutum inoculated with verticillium wilt [72], targeted GhFSD1 and GhFSD2 with sites in intron (Additional file 2), and, GhFSD3 and GhFSD4 were targeted by novel_mir_4246 and m0362 with sites in intron (Fig. 6, Additional file 2). Because we analyzed 
the alternative splicing of $S O D$ genes in G. raimondii and found that there was a basic type of alternative RNA splicing event, intron retention (Additional file 12). So, we conjectured the alternative RNA splicing event existing in $S O D$ genes of upland cotton. Then, the introns had the possibility of being exons erroneously remains in the mature mRNA and encoded amino acids in frame with the neighboring exons. Therefore, we exhibited the predicting miRNA target sites of GhSODs, located at introns (Fig. 6). It was, of course, investigated in further experiments.

In addition, other miRNAs that were predicted to target to GhSODs were also involved in stress responses and biological processes. More concretely, ghr-miR414c, ghr-miR7267, m0081, m0166 and m0362 were involved in cotton fiber differentiation and development [74, 98], and ghr-miR3 regulated the expression of targeted genes during cotton somatic embryogenesis [99]. As a specific well-studied example, miR398 is involved in responses to diverse abiotic and biotic stresses, such as oxidative, salt, heat and ABA, as well as and water, $\mathrm{Cu}$ and phosphate deficiencies, and the additions of sucrose, paraquat, ozone or plant pathogen [63, 73, 100-103]. The mechanisms of miR398 and their targeted genes involved in biotic and abiotic stresses have been widely researched in A. thaliana. miR398 targets two $\mathrm{Cu} / \mathrm{Zn}$ SODs (cytosolic CSD1 and chloroplast-localized CSD2) and the $\mathrm{Cu}$ chaperone for SOD, CCS1, which is activated CSD by delivering a $\mathrm{Cu}$ cofactor to the three $\mathrm{Cu} / \mathrm{Zn}$-SODs. Additionally, miR398 was transcriptionally down-regulated in Arabidopsis under other oxidative stress conditions, and the decreased miR398 level resulted in the accumulation of CSD1 and CSD2 mRNAs, which were important for oxidative stress tolerance [104]. In contrast to the downregulation of miR398 during oxidative stress, an increase in miR398 levels, regulated by the upstream TFs, was observed during $\mathrm{Cu}$ deficiency, leading to the down-regulation of CSD1, CSD2 and CCS1 mRNA expression levels. CCS1 was necessary for the activation of both CSD1 and CSD2 in Arabidopsis; thus, the miR398-guided cleavage of CCS1 mRNA decreased the delivery of $\mathrm{Cu}$ to $\mathrm{CSD}$ and further downregulated CSD activities [71].

To provide a comprehensive model for the role of ghr-miR398 in oxidative stress and nutrient homeostasis, we identified the CCS genes and predicted the targeted sites of miRNAs in the genome of upland cotton (Additional files 2 and 13). Two CCS genes were identified, named GhCCS1 and GhCCS2, which were located in chromosomes $\mathrm{A} 8$ and $\mathrm{D} 8$, respectively. Both ghr-miR398 and novel_mir_1205 targeting GhCCS1 and GhCCS2, respectively, were obtained and the complementary sites were located in the last exon and the 3' UTR. Our results were consistent with corresponding reports in Arabidopsis [71], suggesting that the regulation of the CCS gene by miR398 was conserved in Arabidopsis and upland cotton. However, because of differences in species, growth conditions and treatment methods, the relationships between differential biotic and abiotic stresses, as well as miRNA-mediated stress tolerance in cotton, needs further investigation.

\section{Conclusions}

We identified $18 S O D$ genes, including three types of plant SODs (Cu/Zn-SODs, Mn-SODs and Fe-SODs), that were divided into five subgroups and distributed on 12 of the 26 chromosomes in the upland cotton genome. WGD and polyploidy events, as well as segmental and tandem duplications, contributed to the expansion of the SOD family. Additionally, the complex regulation at the transcriptional level, such as the AS of the pre-mRNA, was a contributory cause of SOD protein diversity. We analyzed the $S O D$ gene family's evolution during the Gossypium and genomic evolution in plants. Based on the results of GO annotations, the SODs of upland cotton were involved in abiotic/biotic stimuli and reproductive development processes, and were regulated by miRNAs. A promoter sequence analysis identified many abiotic/biotic stresses and hormonal-responsive cis-acting elements in the promoter regions of the GhSODs, but different members harbored distinct types and numbers, which indicated that the 18 GhSODs were differentially regulated. Additionally, the expression profiles of the upland cotton $S O D$ gene family were detected using RNA-seq and qPCR data, and suggested that distinct members of the family exhibited different expression patterns in response to abiotic and hormonal stresses, which revealed that GhSODs play roles in different aspects of upland cotton abiotic stress tolerance and hormonal signaling. Moreover, we predicted and analyzed the miRNA-mediated posttranscriptional regulation of the gene family in this species.

Thus, our study helps lay the foundation for further cloning and functional verification of the GhSOD gene family by overexpression and knock down/out using RNAi or genome editing tools, such as CRISPR-Cas9, and provides new insights into the evolution and divergence of the $S O D$ genes in plants. Moreover, these results may increase the understanding of the molecular basis of many important agronomic upland cotton traits, such as fiber development, verticillium and fusarium wilt resistance, and other physiological processes. 


\section{Additional files}

Additional file 1: Table S1. Gene numbers of SOD gene family in 18 plant genomes. (PDF $3335 \mathrm{~kb}$ )

Additional file 2: Table S6. The predictions of GhSODs and GhCCSs targeted by the miRNAs of G. hirsutum. (PDF $3305 \mathrm{~kb}$ )

Additional file 3: Table S7. The details of whole transcriptome sequencing data of G. hirsutumacc. TM. (PDF $3311 \mathrm{~kb}$ )

Additional file 4: Table S8. Gene-specific primers used for quantitative real-time PCR. (PDF 3302 kb)

Additional file 5: Figure S1. The alignment of the genome sequence and mRNA sequence of GhCSD7 with GhCSD8 and its downstream 5000 bp. (PDF 3302 kb)

Additional file 6: Table S2. Motif sequences of GhSOD proteins identified by MEME tools. (PDF 3302 kb)

Additional file 7: Figure S2. Conserved motifs analysis of GhSOD proteins. (PDF $3330 \mathrm{~kb}$ )

Additional file 8: Table S3. The details of sequences used in the phylogenetic relationship analysis. (PDF $3305 \mathrm{~kb}$ )

Additional file 9: Figure S3. Maximum-likelihood (ML) phylogenetic tree constructed for 70 SOD sequences from 9 flagship species and upland cotton. (PDF 3302 kb)

Additional file 10: Table S4. Orthologous $S O D$ gene pairs of $G$. hirsutum, G. arboreum, and G. raimondii. (PDF 3451 kb)

Additional file 11: Table S5. Gene Ontology annotations of 18 upland cotton SOD genes. (PDF $3304 \mathrm{~kb}$ )

Additional file 12: Figure S4. Analysis of alternative splice in 4 GrSOD genes with at least two transcript variants. (PDF 3301 kb)

Additional file 13: The relevant information of upland cotton CCS genes. (PDF $3302 \mathrm{~kb}$ )

\section{Abbreviations}

aa: Amino acid; ABA: Abscisic acid; Amt: Amborella trichopoda; At: Arabidopsis thaliana; Bd: Brachypodium distachyon; bp: base pair; CCS: A cooper chaperone for cooper-zinc superoxide dismutase; CDS: Coding sequence; Cr: Chlamydomonas reinhardtii; CRISPR: Clustered regularly interspaced short palindromic repeats; CSD: Cooper-zinc superoxide dismutase; CZ: Cu/Znsuperoxide dismutase domain; DOA: Day of anthesis; DPA: Days post-anthesis; FPKM: Fragments per kilobase of transcript per million mapped reads; FSD: Iron superoxide dismutase; Ga: Gossypium arboretum; Gh: Gossypium hirsutum; Gm: Glycine max; Gr: Gossypium raimondii; Hv: Hordeum vulgare; IMA: Fe/Mn-SODs, alpha-hairpin domain; IMC: Fe/Mn-SODs, C-terminal domain; kb: Kilobase; Ma: Musa acuminate; miRNA: MicroRNA; MSD: Manganese superoxide dismutase; MW: Theoretical molecular weight of proteins; Mya: Million years ago; NCBI: National Center for Biotechnology Information; nt: Nucleotide; ORF: Open reading frame; Os: Oryza sativa; PCR: Polymerase chain reaction; PCW: Primary cell wall; pl: Isoelectric point; Pp: Physcomitrella patens; Pt: Populus trichocarpa qRT-PCR: Quantitative reverse-transcription polymerase chain reaction; RNAi: RNA interference; ROS: Reactive oxygen species; Sb: Sorghum bicolor; SCW: Secondary cell wall; Si: Setaria italic; SOD: Superoxide dismutases; Ta: Triticum aestivum; TF: Transcription factors; TFBS: Transcription factor binding sites; UBQ: Ubiquitin extension protein; UTR: Untranslated regions; WGD: Whole genome duplication; WGT: Whole genome triplication; ZF: C2H2-type zinc finger domain; Zm: Zea mays

\section{Acknowledgements}

We thank Dr. Zujun Yin of the Institute of Cotton Research, Chinese Academy of Agricultural Sciences, for providing valuable advice on bioinformatics analysis. We also thank reviewers for checking our manuscript and the editors for editing the paper.

\section{Funding}

This research was mainly supported by the China Major Projects for Transgenic Breeding (Grant Nos. 2016ZX08005-004 and 2016ZX08005-002).

\section{Availability of data and materials}

All analysis results data generated during this study were included in this article and its Additional data repository. The genome data supporting the conclusions of this article was from CottonGen (https://www.cottongen.org). The transcriptome sequencing data was from NCBI's Sequence Read Archive (SRA, http://www.ncbi.n/m.nih.gov/sra/) with accession number SRP044705.

\section{Authors' contributions}

WW and FS conceived and designed the research; WW, XZ, FD and RY performed the experiments; $W W$ and $X Z$ analyzed the data and prepared figures; FD and RY contributed analysis tools; WW and XZ wrote the manuscript; FS performed English editing; and all authors reviewed the manuscript. All authors read and approved the final manuscript.

\section{Competing interests}

The authors declare that they have no competing interests.

\section{Consent for publication}

Not applicable.

\section{Ethics approval and consent to participate}

All the cotton materials analyzed for this study were collected from the State Key Laboratory of Crop Biology, Shandong Agricultural University, which were public and available for non-commercial purpose. This article did not contain any studies with human participants or animals performed by any of the authors.

\section{Publisher's Note}

Springer Nature remains neutral with regard to jurisdictional claims in published maps and institutional affiliations.

Received: 22 December 2016 Accepted: 7 May 2017

Published online: 12 May 2017

\section{References}

1. Chen ZJ, Scheffler BE, Dennis E, Triplett BA, Zhang T, Guo W, Chen X, Stelly DM, Rabinowicz PD, Town CD, et al. Toward sequencing cotton (Gossypium) genomes. Plant Physiol. 2007;145(4):1303-10.

2. Gopavajhula VR, Chaitanya KV, Akbar Ali Khan P, Shaik JP, Reddy PN, Alanazi M. Modeling and analysis of soybean (Glycine max. L) Cu/Zn, Mn and Fe superoxide dismutases. Genet Mol Biol. 2013;36(2):225-36.

3. Wang W, Xia MX, Chen J, Yuan R, Deng FN, Shen FF. Gene expression characteristics and regulation mechanisms of superoxide dismutase and its physiological roles in plants under stress. Biochem Mosc. 2016:81(5):465-80.

4. Lin Y-L, Lai Z-X. Superoxide dismutase multigene family in longan somatic embryos: a comparison of CuZn-SOD, Fe-SOD, and Mn-SOD gene structure, splicing, phylogeny, and expression. Mol Breed. 2013;32(3):595-615.

5. Molina-Rueda JJ, Tsai CJ, Kirby EG. The Populus superoxide dismutase gene family and its responses to drought stress in transgenic poplar overexpressing a pine cytosolic glutamine synthetase (GS1a). PLoS One. 2013;8(2):e56421.

6. Feng $X$, Lai Z, Lin Y, Lai G, Lian C. Genome-wide identification and characterization of the superoxide dismutase gene family in Musa acuminata cv. Tianbaojiao (AAA group). BMC Genomics. 2015;16(1):1-16

7. Cannon RE, White JA, Scandalios JG. Cloning of cDNA for maize superoxide dismutase 2 (SOD2). Proc Natl Acad Sci U S A. 1987;84(1):179-83.

8. Zhang F, Li S, Yang S, Wang L, Guo W. Overexpression of a cotton annexin gene, GhAnn 1, enhances drought and salt stress tolerance in transgenic cotton. Plant Mol Biol. 2014:87(1):47-67.

9. Liu Z, Zhang W, Gong X, Zhang Q, Zhou L. A Cu/Zn superoxide dismutase from Jatropha curcas enhances salt tolerance of Arabidopsis thaliana. Genetics and molecular research: GMR. 2014;14(1):2086-98.

10. Kliebenstein DJ, Monde R-A, Last RL. Superoxide dismutase in Arabidopsis: an eclectic enzyme family with disparate regulation and protein localization. Plant Physiol. 1998;118(2):637-50

11. FILIZ E, TOMBULOĞLU H. Genome-wide distribution of superoxide dismutase (SOD) gene families in Sorghum bicolor. Turk J Biol. 2015:39(1):49-59.

12. Wang W, Xia M, Chen J, Deng F, Yuan R, Zhang X, Shen F. Genome-wide analysis of superoxide dismutase gene family in Gossypium raimondii and $G$. arboreum. Plant Gene. 2016;6:18-29. 
13. Voloudakis AE, Marmey P, Delannoy E, Jalloul A, Martinez C, Nicole M. Molecular cloning and characterization of Gossypium hirsutum superoxide dismutase genes during cotton-Xanthomonas campestris pv. malvacearum interaction. Physiol Mol Plant Pathol. 2006;68(4-6):119-27.

14. Payton P, Allen RD, Trolinder N, Scott Holaday A. Over-expression of chloroplast-targeted $\mathrm{Mn}$ superoxide dismutase in cotton (Gossypium hirsutum L., Cv. Coker 312) does not alter the reduction of photosynthesis after short exposures to low temperature and high light intensity. Photosynth Res. 1997;52(3):233-44.

15. Paterson AH, Wendel JF, Gundlach $H$, Guo H, Jenkins J, Jin D, Llewellyn D, Showmaker KC, Shu S, Udall J, et al. Repeated polyploidization of Gossypium genomes and the evolution of spinnable cotton fibres. Nature. 2012;492(7429):423-7.

16. Li F, Fan G, Wang K, Sun F, Yuan Y, Song G, Li Q, Ma Z, Lu C, Zou C, et al. Genome sequence of the cultivated cotton Gossypium arboreum. Nat Genet. 2014;46(6):567-72.

17. Wang K, Wang Z, Li F, Ye W, Wang J, Song G, Yue Z, Cong L, Shang H, Zhu $S$, et al. The draft genome of a diploid cotton Gossypium raimondii. Nat Genet. 2012;44(10):1098-103.

18. Li F, Fan G, Lu C, Xiao G, Zou C, Kohel RJ, Ma Z, Shang H, Ma X, Wu J, et al. Genome sequence of cultivated Upland cotton (Gossypium hirsutum TM-1) provides insights into genome evolution. Nat Biotech. 2015;33(5):524-30.

19. Zhang T, Hu Y, Jiang W, Fang L, Guan X, Chen J, Zhang J, Saski CA, Scheffler BE, Stelly DM, et al. Sequencing of allotetraploid cotton (Gossypium hirsutum L. acc. TM-1) provides a resource for fiber improvement. Nat Biotechnol. 2015:33(5):531-7.

20. Yuan D, Tang Z, Wang M, Gao W, Tu L, Jin X, Chen L, He Y, Zhang L, Zhu L, et al. The genome sequence of Sea-Island cotton (Gossypium barbadense) provides insights into the allopolyploidization and development of superior spinnable fibres. Sci Rep. 2015;5:17662.

21. Liu X, Zhao B, Zheng H-J, Hu Y, Lu G, Yang C-Q, Chen J-D, Chen J-J, Chen D-Y, Zhang L, et al. Gossypium barbadense genome sequence provides insight into the evolution of extra-long staple fiber and specialized metabolites. Sci Rep. 2015;5:14139.

22. Ma T, Wang J, Zhou G, Yue Z, Hu Q, Chen Y, Liu B, Qiu Q, Wang Z, Zhang J, et al. Genomic insights into salt adaptation in a desert poplar. Nat Commun. 2013;4.

23. Camacho C, Coulouris G, Avagyan V, Ma N, Papadopoulos J, Bealer K, Madden TL. BLAST+: architecture and applications. BMC Bioinformatics. 2009; 10:421

24. Quevillon E, Silventoinen V, Pillai S, Harte N, Mulder N, Apweiler R, Lopez R. InterProScan: protein domains identifier. NAR. 2005;33(Web Server issue):W116-20.

25. Finn RD, Bateman A, Clements J, Coggill P, Eberhardt RY, Eddy SR, Heger A, Hetherington K, Holm L, Mistry J, et al. Pfam: the protein families database. Nucleic Acids Res. 2014;42(Database issue):D222-30.

26. Bailey TL, Johnson J, Grant CE, Noble WS. The MEME suite. Nucleic Acids Res. 2015;43(W1):W39-49.

27. Yu CS, Chen YC, Lu CH, Hwang JK. Prediction of protein subcellular localization. Proteins. 2006;64(3):643-51.

28. Horton P, Park K-J, Obayashi T, Fujita N, Harada H, Adams-Collier CJ, Nakai K WOLF PSORT: protein localization predictor. NAR. 2007;35 suppl 2:W585-7.

29. Thompson JD, Gibson TJ, Higgins DG, et al. Multiple sequence alignment using ClustalW and ClustalX. In: Baxevanis AD, editor. Current protocols in bioinformatics. 2002. Chapter 2:Unit 23.

30. Talavera G, Castresana J. Improvement of phylogenies after removing divergent and ambiguously aligned blocks from protein sequence alignments. Syst Biol. 2007;56(4):564-77.

31. Keane TM, Creevey CJ, Pentony MM, Naughton TJ, McLnerney JO. Assessment of methods for amino acid matrix selection and their use on empirical data shows that ad hoc assumptions for choice of matrix are not justified. BMC Evol Biol. 2006;6:29.

32. Kumar S, Nei M, Dudley J, Tamura K. MEGA: a biologist-centric software for evolutionary analysis of DNA and protein sequences. Brief Bioinform. 2008;9(4):299-306

33. Krzywinski M, Schein J, Birol I, Connors J, Gascoyne R, Horsman D, Jones SJ, Marra MA. Circos: an information aesthetic for comparative genomics. Genome Res. 2009;19(9):1639-45.

34. Lee TH, Tang H, Wang X, Paterson AH. PGDD: a database of gene and genome duplication in plants. Nucleic Acids Res. 2013;41(Database issue):D1152-8.
35. Altschul SF, Gish W, Miller W, Myers EW, Lipman DJ. Basic local alignment search tool. J Mol Biol. 1990;215(3):403-10.

36. Wang $Y$, Tang H, Debarry JD, Tan $X$, Li J, Wang $X$, Lee TH, Jin H, Marler B, Guo H, et al. MCScanX: a toolkit for detection and evolutionary analysis of gene synteny and collinearity. Nucleic Acids Res. 2012:40(7), e49.

37. Du D, Hao R, Cheng T, Pan H, Yang W, Wang J, Zhang Q. Genome-wide analysis of the AP2/ERF gene family in Prunus mume. Plant Mol Biol Rep. 2013;31(3):741-50.

38. Hu B, Jin J, Guo A-Y, Zhang H, Luo J, Gao G. GSDS 2.0: an upgraded gene feature visualization server. Bioinformatics. 2015;31(8):1296-7.

39. Ashburner M, Ball CA, Blake JA, Botstein D, Butler H, Cherry JM, Davis AP, Dolinski K, Dwight SS, Eppig JT, et al. Gene Ontology: tool for the unification of biology. Nat Genet. 2000;25(1):25-9.

40. Ye J, Fang L, Zheng H, Zhang Y, Chen J, Zhang Z, Wang J, Li S, Li R, Bolund $L$, et al. WEGO: a web tool for plotting GO annotations. Nucleic Acids Res. 2006:34(Web Server issue):W293-7.

41. Kozomara A, Griffiths-Jones S. miRBase: annotating high confidence microRNAs using deep sequencing data. Nucleic Acids Res. 2014;42(Database issue):D68-73.

42. Zhang Z, Yu J, Li D, Zhang Z, Liu F, Zhou X, Wang T, Ling $Y$, Su Z. PMRD: plant microRNA database. Nucleic Acids Res. 2010;38(Database issue):D806-13.

43. Dai $X$, Zhao PX. psRNATarget: a plant small RNA target analysis server. Nucleic Acids Res. 2011;39(Web Server issue):W155-9.

44. Lescot M, Dehais $P$, Thijs $G$, Marchal $K$, Moreau $Y$, Van de Peer $Y$, Rouze $P$, Rombauts S. PlantCARE, a database of plant cis-acting regulatory elements and a portal to tools for in silico analysis of promoter sequences. Nucleic Acids Res. 2002;30(1):325-7.

45. Kim D, Langmead B, Salzberg SL. HISAT: a fast spliced aligner with low memory requirements. Nat Methods. 2015;12(4):357-60.

46. Li H, Handsaker B, Wysoker A, Fennell T, Ruan J, Homer N, Marth G, Abecasis $G$, Durbin R. Genome project data processing $S$ : the sequence alignment/Map format and SAMtools. Bioinformatics. 2009;25(16):2078-9.

47. Pertea M, Pertea GM, Antonescu CM, Chang T-C, Mendell JT, Salzberg SL. StringTie enables improved reconstruction of a transcriptome from RNA-seq reads. Nat Biotech. 2015;33(3):290-5.

48. Schmittgen TD, Livak KJ. Analyzing real-time PCR data by the comparative C(T) method. Nat Protoc. 2008;3(6):1101-8.

49. Singh VK, Mangalam AK, Dwivedi S, Naik S. Primer premier: program for design of degenerate primers from a protein sequence. BioTechniques. 1998;24(2):318-9.

50. Dehury B, Sarma K, Sarmah R, Sahu J, Sahoo S, Sahu M, Sen P, Modi M, Sharma G, Choudhury M, et al. In silico analyses of superoxide dismutases (SODs) of rice (Oryza sativa L.). J Plant Biochem Biotechnol. 2013;22(1):150-6.

51. Guruprasad K, Reddy BVB, Pandit MW. Correlation between stability of a protein and its dipeptide composition: a novel approach for predicting in vivo stability of a protein from its primary sequence. Protein Eng. 1990;4(2):155-61.

52. Hu G-h, Yu S-x, Fan S-I, Song M-Z. Cloning and expressing of a gene encoding cytosolic CopperEinc superoxide dismutase in the upland cotton. Agric Sci Chin. 2007:6(5):536-44.

53. Zeng X-C, Liu Z-G, Shi P-H, Xu Y-Z, Sun J, Fang Y, Yang G, Wu J-Y, Kong D-J, Sun W-C. Cloning and expression analysis of copper and zinc superoxide dismutase (Cu/Zn-SOD) gene from Brassica campestris L. Acta Agron Sin. 2014:40(4):636-43

54. Corpas FJ, Fernández-Ocaña A, Carreras A, Valderrama R, Luque F, Esteban FJ, Rodríguez-Serrano M, Chaki M, Pedrajas JR, Sandalio LM. The expression of different superoxide dismutase forms is cell-type dependent in olive (Olea europaea L.) leaves. Plant Cell Physiol. 2006:47(7):984-94.

55. Pan Y, Wu LJ, Yu ZL. Effect of salt and drought stress on antioxidant enzymes activities and SOD isoenzymes of liquorice (Glycyrrhiza uralensis Fisch). Plant Growth Regul. 2006:49(2):157-65.

56. Fink RC, Scandalios JG. Molecular evolution and structure-function relationships of the superoxide dismutase gene families in angiosperms and their relationship to other eukaryotic and prokaryotic superoxide dismutases. Arch Biochem Biophys. 2002;399(1):19-36.

57. Smith MW, Doolittle RF. A comparison of evolutionary rates of the two major kinds of superoxide dismutase. J Mol Evol. 1992;34(2):175-84. 
58. Xu L, Zhu L, Tu L, Liu L, Yuan D, Jin L, Long L, Zhang X. Lignin metabolism has a central role in the resistance of cotton to the wilt fungus Verticillium dahliae as revealed by RNA-Seq-dependent transcriptional analysis and histochemistry. J Exp Bot. 2011;62(15):5607-21.

59. Hirsh $A E$, Fraser HB. Protein dispensability and rate of evolution. Nature. 2001:411(6841):1046-9.

60. Innan H, Kondrashov F. The evolution of gene duplications: classifying and distinguishing between models. Nat Rev Genet. 2010;11(2):97-108.

61. Bindschedler LV, Palmblad M, Cramer R. Hydroponic isotope labelling of entire plants (HILEP) for quantitative plant proteomics; an oxidative stress case study. Phytochemistry. 2008;69(10):1962-72.

62. Jagadeeswaran G, Saini A, Sunkar R. Biotic and abiotic stress down-regulate miR398 expression in Arabidopsis. Planta. 2009;229(4):1009-14.

63. Jia X, Wang WX, Ren L, Chen QJ, Mendu V, Willcut B, Dinkins R, Tang X, Tang G. Differential and dynamic regulation of miR398 in response to ABA and salt stress in Populus tremula and Arabidopsis thaliana. Plant Mol Biol. 2009:71(1-2):51-9.

64. Zhou ZS, Zeng HQ, Liu ZP, Yang ZM. Genome-wide identification of Medicago truncatula microRNAs and their targets reveals their differential regulation by heavy metal. Plant Cell Environ. 2012;35(1):86-99.

65. Kurepa J, Van Montagu M, Inzé D. Expression of sodC $p$ and $\operatorname{sod} B$ genes in Nicotiana tabacum: effects of light and copper excess. J Exp Bot. 1997:48(12):2007-14.

66. Gupta AS, Heinen JL, Holaday AS, Burke JJ, Allen RD. Increased resistance to oxidative stress in transgenic plants that overexpress chloroplastic $\mathrm{Cu} / \mathrm{Zn}$ superoxide dismutase. Proc Natl Acad Sci U S A. 1993;90(4):1629-33.

67. Su L-T, Wang Y, Liu D-Q, Li X-W, Zhai Y, Sun X, Li X-Y, Liu Y-J, Li J-W, Wang Q-Y. The soybean gene, GmMYBJ2, encodes a R2R3-type transcription factor involved in drought stress tolerance in Arabidopsis thaliana. Acta Physiol Plant. 2015;37(7):1-12.

68. Fang W, Ding W, Zhao X, Zhang F, Gao S, Li X, Xiao K. Expression profile and function characterization of the MYB type transcription factor genes in wheat (Triticum aestivum L.) under phosphorus deprivation. Acta Physiol Plant. 2015;38(1):1-13.

69. Pérez-Díaz JR, Pérez-Díaz J, Madrid-Espinoza J, González-Villanueva E, Moreno Y, Ruiz-Lara S. New member of the R2R3-MYB transcription factors family in grapevine suppresses the anthocyanin accumulation in the flowers of transgenic tobacco. Plant Mol Biol. 2016;90(1):63-76.

70. Yu Y-T, Wu Z, Lu K, Bi C, Liang S, Wang X-F, Zhang D-P. Overexpression of the MYB transcription factor MYB28 or MYB99 confers hypersensitivity to abscisic acid in arabidopsis. J Plant Biol. 2016;59(2):152-61.

71. Beauclair $L, Y u A$, Bouche N. microRNA-directed cleavage and translational repression of the copper chaperone for superoxide dismutase mRNA in Arabidopsis. Plant J. 2010;62(3):454-62.

72. Zhang Y, Wang W, Chen J, Liu J, Xia M, Shen F. Identification of miRNAs and their targets in cotton inoculated with Verticillium dahliae by high-throughput sequencing and degradome analysis. Int J Mol Sci. 2015;16(7):14749.

73. Guan Q, Lu X, Zeng H, Zhang Y, Zhu J. Heat stress induction of miR398 triggers a regulatory loop that is critical for thermotolerance in Arabidopsis. PIJ. 2013;74(5):840-51.

74. Li Q, Jin X, Zhu YX. Identification and analyses of miRNA genes in allotetraploid Gossypium hirsutum fiber cells based on the sequenced diploid G. raimondii genome. J Genet Genomics. 2012;39(7):351-60.

75. Qiu CX, Xie FL, Zhu YY, Guo K, Huang SQ, Nie L, Yang ZM. Computational identification of microRNAs and their targets in Gossypium hirsutum expressed sequence tags. Gene. 2007;395(1-2):49-61.

76. Qin Y-M, Zhu Y-X. How cotton fibers elongate: a tale of linear cell-growth mode. Curr Opin Plant Biol. 2011;14(1):106-11.

77. Jackson S, Chen ZJ. Genomic and expression plasticity of polyploidy Curr Opin Plant Biol. 2010;13(2):153-9.

78. Jiao Y, Li J, Tang H, Paterson AH. Integrated syntenic and phylogenomic analyses reveal an ancient genome duplication in monocots. Plant Cell. 2014:7:2792-802

79. Renny-Byfield S, Gallagher JP, Grover CE, Szadkowski E, Page JT, Udall JA Wang X, Paterson AH, Wendel JF. Ancient gene duplicates in Gossypium (cotton) exhibit near-complete expression divergence. Genome Biol Evol. 2014:6(3):559-71.

80. Katyshev Al, Konstantinov YM, Kobzev VF. Characterization of Mn-and $\mathrm{Cu} / \mathrm{Zn}$-containing superoxide dismutase gene transcripts in Larix gmelinii. Mol Biol. 2006;40(2):327-9.
81. Feng $W$, Hongbin W, Bing L, Jinfa W. Cloning and characterization of a novel splicing isoform of the iron-superoxide dismutase gene in rice (Oryza sativa L.). Plant Cell Rep. 2006;24(12):734-42.

82. Baek KH, Skinner DZ, Ling P, Chen X. Molecular structure and organization of the wheat genomic manganese superoxide dismutase gene. Genome. 2006:49(3):209-18

83. Kitagawa N, Washio T, Kosugi S, Yamashita T, Higashi K, Yanagawa H, Higo K, Satoh K, Ohtomo Y, Sunako T, et al. Computational analysis suggests that alternative first exons are involved in tissue-specific transcription in rice (Oryza sativa). Bioinformatics. 2005;21(9):1758-63.

84. Wendel JF. New World tetraploid cottons contain Old World cytoplasm. Proc Natl Acad Sci U S A. 1989;86(11):4132-6.

85. Schnable JC, Springer NM, Freeling M. Differentiation of the maize subgenomes by genome dominance and both ancient and ongoing gene loss. Proc Natl Acad Sci U S A. 2011:108(10):4069-74.

86. Senchina DS, Alvarez I, Cronn RC, Liu B, Rong J, Noyes RD, Paterson AH, Wing RA, Wilkins TA, Wendel JF. Rate variation among nuclear genes and the Age of polyploidy in Gossypium. Mol Biol Evol. 2003;20(4):633-43.

87. Berkner LV, Marshall LC. On the origin and rise of oxygen concentration in the Earth's atmosphere. J Atmos Sci. 1965;22(3):225-61.

88. Blankenship RE. Origin and early evolution of photosynthesis. Photosynth Res. 1992:33(2):91-111.

89. Cheng F, Sun C, Wu J, Schnable J, Woodhouse MR, Liang J, Cai C, Freeling $M$, Wang $X$. Epigenetic regulation of subgenome dominance following whole genome triplication in Brassica rapa. New Phytol. 2016;211(1):288-99.

90. Gill S, Anjum N, Gill R, Yadav S, Hasanuzzaman M, Fujita M, Mishra P, Sabat S, Tuteja N. Superoxide dismutase-mentor of abiotic stress tolerance in crop plants. Environ Sci Pollut Res. 2015;22(14):10375-94.

91. Tsang EW, Bowler C, Herouart D, Van Camp W, Villarroel R, Genetello C, Van Montagu M, Inze D. Differential regulation of superoxide dismutases in plants exposed to environmental stress. Plant Cell. 1991:3(8):783-92.

92. He Q, Jones DC, Li W, Xie F, Ma J, Sun R, Wang Q, Zhu S, Zhang B. Genome-wide identification of R2R3-MYB genes and expression analyses during abiotic stress in Gossypium raimondii. Sci Rep. 2016;6:22980.

93. Salih H, Gong W, He S, Sun G, Sun J, Du X. Genome-wide characterization and expression analysis of MYB transcription factors in Gossypium hirsutum. BMC Genet. 2016:17(1):1-12.

94. Yang A, Dai X, Zhang W-H. A R2R3-type MYB gene, OsMYB2, is involved in salt, cold, and dehydration tolerance in rice. J Exp Bot. 2012;63(7):2541-56

95. Ganesan G, Sankararamasubramanian HM, Harikrishnan M, Ashwin G Parida A. A MYB transcription factor from the grey mangrove is induced by stress and confers $\mathrm{NaCl}$ tolerance in tobacco. J Exp Bot. 2012;63(12):4549-61.

96. Kim HJ, Kato N, Kim S, Triplett B. Cu/Zn superoxide dismutases in developing cotton fibers: evidence for an extracellular form. Planta. 2008;228(2):281-92.

97. Ergun S, Oztuzcu S. Sequence-based analysis of 5'UTR and coding regions of CASP3 in terms of miRSNPs and SNPs in targetting miRNAs. Comput Biol Chem. 2016:62:70-4

98. Zhang B, Wang Q, Wang K, Pan X, Liu F, Guo T, Cobb GP, Anderson TA. Identification of cotton microRNAs and their targets. Gene. 2007;397(1):26-37

99. Yang X, Wang L, Yuan D, Lindsey K, Zhang X. Small RNA and degradome sequencing reveal complex miRNA regulation during cotton somatic embryogenesis. J Exp Bot. 2013;64(6):1521-36.

100. Naya L, Paul S, Valdes-Lopez O, Mendoza-Soto AB, Nova-Franco B, Sosa-Valencia G, Reyes JL, Hernandez G. Regulation of copper homeostasis and biotic interactions by microRNA 398b in common bean. PLoS One. 2014;9(1):e84416.

101. Jovanovic Z, Stanisavljevic N, Mikic A, Radovic S, Maksimovic V. Water deficit down-regulates miR398 and miR408 in pea (Pisum sativum L.). Plant Physiol Biochem. 2014:83:26-31.

102. Saenen E, Horemans N, Vanhoudt N, Vandenhove H, Biermans G, Van Hees M, Wannijn J, Vangronsveld J, Cuypers A. MiRNA398b and miRNA398c are involved in the regulation of the SOD response in uranium-exposed Arabidopsis thaliana roots. Environ Exp Bot. $2015 ; 116: 12-9$ 
103. Lu Y, Feng Z, Bian L, Xie H, Liang J. miR398 regulation in rice of the responses to abiotic and biotic stresses depends on CSD1 and CSD2 expression. Funct Plant Biol. 2010;38(1):44-53.

104. Sunkar R, Kapoor A, Zhu J-K. Posttranscriptional induction of two Cu/Zn superoxide dismutase genes in Arabidopsis is mediated by downregulation of miR398 and important for oxidative stress tolerance. The Plant Cell Online. 2006;18(8):2051-65.

Submit your next manuscript to BioMed Central and we will help you at every step:

- We accept pre-submission inquiries

- Our selector tool helps you to find the most relevant journal

- We provide round the clock customer support

- Convenient online submission

- Thorough peer review

- Inclusion in PubMed and all major indexing services

- Maximum visibility for your research

Submit your manuscript at www.biomedcentral.com/submit 